\title{
RATIONALITY AND POINCARÉ FAMILIES FOR VECTOR BUNDLES WITH EXTRA STRUCTURE ON A CURVE
}

\author{
NORBERT HOFFMANN
}

\begin{abstract}
Iterated Grassmannian bundles over moduli stacks of vector bundles on a curve are shown to be birational to an affine space times a moduli stack of degree 0 vector bundles, following the method of King and Schofield. Applications include the birational type of some Brill-Noether loci, of moduli schemes for vector bundles with parabolic structure or with level structure and for A. Schmitt's decorated vector bundles. A further consequence concerns the existence of Poincaré families on finite coverings of the moduli schemes.
\end{abstract}

\section{INTRODUCTION}

Let $C$ be a smooth projective algebraic curve of genus $g \geq 2$, say over an algebraically closed field $k$, and let $L$ be a line bundle on $C$. A. King and A. Schofield 21] have proved that the coarse moduli scheme $\mathfrak{B u n}_{r, L}$ of stable vector bundles $E$ on $C$ with $\operatorname{rk}(E)=r$ and $\operatorname{det}(E) \cong L$ is rational if the highest common factor $h$ of $r$ and $\operatorname{deg}(L)$ is 1 . The present paper generalises this result to vector bundles with some extra structure, e.g. with parabolic structure in the sense of 30. In that case, we obtain that the moduli scheme is rational if the highest common factor $h$ of rank, degree and all multiplicities is 1, improving results of [5].

This highest common factor $h$ also governs the existence of Poincaré families: Standard methods [32. Chapter 4, §5] construct e. g. a Poincaré family on the coarse moduli scheme of stable parabolic vector bundles if $h=1$, whereas Ramanan 34 has proved that there is no Poincaré family on any open subscheme of $\mathfrak{B u n} \mathfrak{n}_{r, L}$ if $h \neq 1$. We provide here a common source for such results on the rationality of coarse moduli schemes and on the existence of Poincaré families on them: Our main theorem 5.1 states that the moduli stack of fixed determinant vector bundles with extra structure is birational to an affine space $\mathbb{A}^{s}$ times a moduli stack $\mathcal{B} u n_{h, L_{0}}$ of rank $h$ vector bundles with fixed determinant $L_{0}$ of degree 0 .

The proof of this theorem requires to carefully keep track of something as inconspicuous as the scalar automorphisms of the vector bundles $E$ on $C$. In some sense, this is already implicit in the King-Schofield proof, namely in their notion of weight [21, p. 526] and in their Brauer class $\psi_{r, d}$ - which can be interpreted as the obstruction against a Poincaré family. But the (admittedly more abstract) stack language helps to clarify and generalise here, for example to arbitrary infinite base field $k$ (as long as $C$ has a $k$-rational point). Apart from that, we basically follow the method of [21]: The moduli stack $\mathcal{B} u n_{r, L}$ is shown to be birational to a

2000 Mathematics Subject Classification. Primary: 14H60; Secondary: 14E08.

Key words and phrases. vector bundles on curves, moduli space, rationality. 
Grassmannian bundle over $\mathcal{B} u n_{r_{1}, L_{1}}$ for some $r_{1}<r$ if $r$ does not divide $\operatorname{deg}(L)$; then induction is used.

A new ingredient in our proof is the refinement 3.13 of Ramanan's theorem mentioned above; it allows to extend our results to vector bundles with extra structures parameterised by iterated Grassmannian bundles over $\mathcal{B} u n_{r, L}$, and it also yields that finite coverings of the coarse moduli scheme can only admit Poincaré families if their degree is a multiple of $h$, cf. corollary 5.3

The structure of this text is as follows: Section 1 presents the moduli stacks that this paper deals with, and collects some basic information about them. In section 2] we introduce the notions of $\mathbb{G}_{m}$-stack and of $\mathbb{G}_{m}$-gerbe in order to systematically distinguish 'scalar automorphisms'. Section 3 and section 4 deal with vector bundles and the associated Grassmannian bundles on such stacks, respectively. Finally, section 5 contains statement and proof of the main theorem as well as some consequences and examples, including vector bundles with parabolic structure, Brill-Noether loci and A. Schmitt's decorated vector bundles.

I wish to thank J. Heinloth for numerous explanations and fruitful discussions about these moduli stacks, in particular for his help in proving 3.13] and 3.8 I also thank the Tata Institute of Fundamental Research in Bombay for support and hospitality while this text was written.

\section{Moduli stacks of Vector Bundles on a CURVE}

Let $k$ be an infinite field, and let $C$ be a geometrically irreducible, smooth projective curve over $k$ of genus $g \geq 2$ which has a rational point $P \in C(k)$. This paper deals with moduli spaces of vector bundles $E$ on $C$ from a birational point of view.

Remark 1.1. Usually, such questions have been studied over algebraically closed fields $k$ or even over $k=\mathbb{C}$. Working over more general base fields does not make a big difference for our arguments due to lemma 1.5 below, but it has some minor technical advantages; see for example remark 1.4 ii.

By an algebraic stack $\mathcal{M}$ over $k$, we always mean an Artin stack $\mathcal{M}$ that is locally of finite type over $k$ (but not necessarily quasi-compact); a standard reference for these notions is 26. Recall that $\mathcal{M}$ can be given by a groupoid $\mathcal{M}(S)$ for each $k$ scheme $S$, a functor $f^{*}: \mathcal{M}(S) \rightarrow \mathcal{M}(T)$ for each morphism of $k$-schemes $f: T \rightarrow S$ and isomorphisms of functors $(f \circ g)^{*} \cong g^{*} \circ f^{*}$. Such stacks over $k$ form a 2-category: A 1-morphism $\Phi: \mathcal{M} \rightarrow \mathcal{M}^{\prime}$ can be given by functors $\Phi(S): \mathcal{M}(S) \rightarrow \mathcal{M}^{\prime}(S)$ and isomorphisms of functors $f^{*} \circ \Phi(S) \cong \Phi(T) \circ f^{*}$; a 2-morphism $\tau: \Phi_{1} \Rightarrow \Phi_{2}$ can be given by natural transformations $\tau(S): \Phi_{1}(S) \Rightarrow \Phi_{2}(S)$. In all this, one can replace the base field $k$ more generally by a noetherian $\operatorname{ring} A$.

Two irreducible algebraic stacks $\mathcal{M}$ and $\mathcal{M}^{\prime}$ over $k$ are called birational if some open substacks $\emptyset \neq \mathcal{U} \subseteq \mathcal{M}$ and $\emptyset \neq \mathcal{U}^{\prime} \subseteq \mathcal{M}^{\prime}$ are 1-isomorphic, and stably birational if $\mathbb{A}^{s} \times \mathcal{M}$ and $\mathbb{A}^{s^{\prime}} \times \mathcal{M}^{\prime}$ are birational for some $s, s^{\prime} \in \mathbb{N}$.

Example 1.2. We denote by $\mathcal{B u n}_{r, d}$ the moduli stack of vector bundles $E$ on $C$ with rank $r \geq 1$ and degree $d \in \mathbb{Z}$. More precisely, $\mathcal{B} u n_{r, d}(S)$ is the groupoid of all vector bundles $\mathcal{E}$ on $C \times{ }_{k} S$ with rank $r$ and degree $d$ over every geometric point of $S$, and $f^{*}: \mathcal{B} u n_{r, d}(S) \rightarrow \mathcal{B} u n_{r, d}(T)$ is 'the' usual pull-back functor.

Example 1.3. Given a line bundle $L$ of degree $d$ on $C$, we denote by $\mathcal{B} u n_{r, L} \subseteq \mathcal{B} u n_{r, d}$ the (reduced) closed substack of vector bundles $E$ on $C$ with $\operatorname{det}(E) \cong L$. More 
precisely, $\mathcal{B u n}_{r, L}(S) \subseteq \mathcal{B} u n_{r, d}(S)$ is the full subgroupoid of all vector bundles $\mathcal{E}$ on $C \times{ }_{k} S$ for which $\operatorname{det}(\mathcal{E})$ is Zariski-locally in $S$ isomorphic to the pull-back of $L$.

The basic properties of the algebraic stacks $\mathcal{B} u n_{r, d}$ and $\mathcal{B} u n_{r, L}$ can be found for example in 11, [16] or the appendix of 18]: They are smooth over $k$ by standard deformation theory, $\operatorname{dim}_{k} \mathcal{B} u n_{r, d}=r^{2}(g-1)$ and $\operatorname{dim}_{k} \mathcal{B} u n_{r, L}=r^{2}(g-1)-g$. Moreover, they are geometrically connected, hence geometrically irreducible.

Remarks 1.4. i) Sending a vector bundle $E$ on $C$ to the line bundle $\operatorname{det}(E)$ defines a smooth morphism det : $\mathcal{B u n}_{r, d} \rightarrow \operatorname{Pic}^{d}(C)$ to the Picard scheme of $C$. Its fibre over the $k$-point given by a line bundle $L$ of degree $d$ on $C$ is precisely $\mathcal{B} u n_{r, L}$.

ii) Let $K=k\left(\operatorname{Pic}^{d}(C)\right)$ be the function field, and let the line bundle $\mathcal{L}$ on $C \otimes_{k} K$ be the generic fibre of a Poincaré family. Then the moduli stack $\mathcal{B} u n_{r, \mathcal{L}}$ over $K$ is precisely the generic fibre of det. Thus birational results for fixed degree moduli spaces follow from their analogues for fixed determinant moduli spaces; this allows us to concentrate on the latter in the sequel.

Lemma 1.5. Every open substack $\emptyset \neq \mathcal{U} \subseteq \mathcal{B} u n_{r, L}$ contains a $k$-rational point.

Proof. This follows from the well-known unirationality of $\mathcal{B} u n_{r, L}$. More precisely:

We consider the open substack in $\mathcal{B} u n_{r, L}$ of vector bundles $E$ with $\mathrm{H}^{1}(E)=0$ which are generated by $\mathrm{H}^{0}(E)$. Twisting by a sufficiently ample line bundle on $C$, we may assume that it is nonempty; we may even assume that it contains $\mathcal{U}$ by shrinking the latter.

Now let $U \subseteq \operatorname{Hom}\left(L^{\text {dual }}, \mathcal{O}_{C}^{r+1}\right)$ be the open subscheme of nonzero morphisms $\varphi: L^{\text {dual }} \rightarrow \mathcal{O}_{C}^{r+1}$ whose cokernel is torsionfree. Sending $\varphi$ to its cokernel defines a morphism $\Phi: U \rightarrow \mathcal{B u n}_{r, L}$; we claim that its image contains $\mathcal{U}$. This implies that $\Phi^{-1}(\mathcal{U})$ is nonempty and open in the affine space $\operatorname{Hom}\left(L^{\text {dual }}, \mathcal{O}_{C}^{r+1}\right)$ over $k$, so it contains a $k$-rational point; its image under $\Phi$ is then a $k$-rational point in $\mathcal{U}$.

To prove the claim, we may assume that $k$ is algebraically closed. Let $E$ be a rank $r$ vector bundle on $C$ which is generated by $\mathrm{H}^{0}(E)$; it suffices to construct a surjection $\mathcal{O}_{C}^{r+1} \rightarrow E$ since its kernel will be isomorphic to $\operatorname{det}(E)^{\text {dual }}$. We follow an argument of Ramanan [34, Lemma 3.1] who attributes the result to Atiyah.

Let $\Delta \subseteq C \times \mathrm{H}^{0}(E)^{r+1}$ be the closed subscheme where the $r+1$ universal sections do not generate $E$. The restriction $\Delta_{x}$ of $\Delta$ to a point $x \in C(k)$ is the inverse image under the surjective evaluation map $\mathrm{H}^{0}(E)^{r+1} \rightarrow E_{x}^{r+1}$ of all $r+1$-tuples that do not generate the fibre $E_{x} \cong k^{r}$; by linear algebra, the latter has codimension 2 in $E_{x}^{r+1}$, so $\Delta_{x}$ has codimension 2 in $\mathrm{H}^{0}(E)^{r+1}$. Thus $\Delta$ has codimension 2 in $C \times \mathrm{H}^{0}(E)^{r+1}$, so its projection to $\mathrm{H}^{0}(E)^{r+1}$ has codimension $\geq 1$. This shows that there is indeed a surjection $\mathcal{O}_{C}^{r+1} \rightarrow E$.

Remark 1.6. The above lemma implies that $k$-points are dense in $\mathcal{B} u n_{r, d}$ if and only if they are dense in the Jacobian $J_{C}=\operatorname{Pic}^{0}(C)$. The latter is easily seen to hold if $C(k)$ is infinite. On the other hand, an example with $J_{C}(k)$ finite due to Faddeev [10] is the Fermat curve $C \subseteq \mathbb{P}^{2}$ with equation $x^{5}+y^{5}=z^{5}$ over $k=\mathbb{Q}$.

Remark 1.7. The same arguments as for $\mathcal{B u n}_{r, d}$ show in fact that the moduli stack $\mathcal{C}_{o} h_{r, d}$ of coherent sheaves $F$ on $C$ with rank $r$ and degree $d$ is algebraic, smooth of dimension $(g-1) r^{2}$ over $k$ and irreducible; cf. for example the appendix of [18. One still has a determinant morphism det $: \mathcal{C}_{o} h_{r, d} \rightarrow \operatorname{Pic}^{d}(C)$, e. g. by [20, p. 37].

This includes in particular the case $r=0$ and $d \geq 0$, whereas of course $\mathcal{C}_{0} h_{0, d}=\emptyset$ for $d<0$. If $r \geq 1$, then $\emptyset \neq \mathcal{B} u n_{r, d} \subseteq \mathcal{C}_{o} h_{r, d}$ is an open substack, thus dense. 
The following further examples of algebraic stacks will also be used in the sequel.

Example 1.8. We denote by $\mathcal{V} \mathrm{ct}_{n}$ the moduli stack of $n$-dimensional vector spaces over $k$; more precisely, $\mathcal{V e c t}_{n}(S)$ is the groupoid of rank $n$ vector bundles on $S$. Vect $_{n}$ is 1-isomorphic to the stack quotient $\mathrm{BGL}_{n}$ of $\operatorname{Spec}(k)$ modulo the trivial action of $\mathrm{GL}_{n}$; thus it is algebraic, smooth of dimension $-n^{2}$ over $k$ and irreducible.

Example 1.9. Let $A$ be a finitely generated $k$-algebra, and let $F_{\nu}$ be for $\nu=1,2$ a coherent sheaf on $C \times{ }_{k} \operatorname{Spec}(A)$ which is flat over $A$ and whose restriction to each point of $\operatorname{Spec}(A)$ has rank $r_{\nu}$ and degree $d_{\nu}$. We denote by $\operatorname{Ext}\left(F_{2}, F_{1}\right)$ the moduli stack over $A$ of extensions of $F_{2}$ by $F_{1}$; more precisely, $\mathcal{E} x t\left(F_{2}, F_{1}\right)(S)$ is the following groupoid for each $A$-scheme $p: S \rightarrow \operatorname{Spec}(A)$ :

- Its objects are the exact sequence $0 \rightarrow p^{*} F_{1} \rightarrow \mathcal{F} \rightarrow p^{*} F_{2} \rightarrow 0$ of coherent sheaves on $C \times_{k} S$; note that $\mathcal{F}$ is automatically flat over $S$.

- Its morphisms are the $\mathcal{O}_{C \times_{k} S}$-module isomorphisms $\mathcal{F} \rightarrow \mathcal{F}^{\prime}$ that are compatible with the identity on $p^{*} F_{\nu}$ for $\nu=1,2$.

Lemma 1.10. $\mathcal{E} x t\left(F_{2}, F_{1}\right)$ is algebraic, of finite type and smooth of relative dimension $(g-1) r_{1} r_{2}+r_{1} d_{2}-r_{2} d_{1}$ over $A$, with all its fibers geometrically irreducible.

Proof. Using [20, Prop. 2.1.10], we can find an injective morphism $i: E^{\prime} \hookrightarrow E$ of vector bundles on $C \times_{k} \operatorname{Spec}(A)$ with cokernel $F_{2}$. One checks immediately that

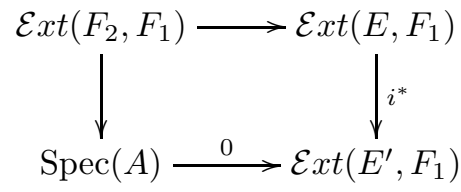

is a 1 -cartesian diagram; here 0 is given by the trivial extension $F_{1} \oplus E^{\prime}$.

We choose an open affine covering $X=U \cup V$ and put $U_{A}:=U \times_{k} \operatorname{Spec}(A)$, $V_{A}:=V \times_{k} \operatorname{Spec}(A)$. This yields a Čech complex

$C^{\bullet}\left(E, F_{1}\right)=\operatorname{Hom}\left(\left.E\right|_{U_{A}},\left.F_{1}\right|_{U_{A}}\right) \oplus \operatorname{Hom}\left(\left.E\right|_{V_{A}},\left.F_{1}\right|_{V_{A}}\right) \stackrel{\delta}{\longrightarrow} \operatorname{Hom}\left(\left.E\right|_{U_{A} \cap V_{A}},\left.F_{1}\right|_{U_{A} \cap V_{A}}\right)$

whose differential $\delta$ sends two morphisms $\alpha_{U}$ over $U_{A}$ and $\alpha_{V}$ over $V_{A}$ to $\alpha_{U}-\alpha_{V}$ over $U_{A} \cap V_{A}$. We denote the associated Picard stack [15. Exp. XVIII, 1.4] by $C^{1}\left(E, F_{1}\right) / C^{0}\left(E, F_{1}\right)$; this is by definition the stack generated by the prestack $\mathcal{M}$ consisting of the following groupoid $\mathcal{M}(S)$ for each $A$-scheme $p: S \rightarrow \operatorname{Spec}(A)$ :

- Its objects are the 1 -cochains $\varphi \in C^{1}\left(E, F_{1}\right) \otimes_{A} \Gamma\left(S, \mathcal{O}_{S}\right)$.

- Its morphisms $\alpha: \varphi \rightarrow \psi$ are the 0-cochains $\alpha \in C^{0}\left(E, F_{1}\right) \otimes_{A} \Gamma\left(S, \mathcal{O}_{S}\right)$ with $\delta(\alpha)=\psi-\varphi$.

- Its composition law is the addition of 0 -cochains.

If an object $\varphi$ of $\mathcal{M}(S)$ is given, then id $+\varphi$ is an automorphism of the trivial extension $p^{*} F_{1} \oplus p^{*} E$ over $(U \cap V) \times_{k} S$; using it to glue the trivial extensions over $U \times_{k} S$ and over $V \times_{k} S$, we obtain an object of $\mathcal{E} x t\left(E, F_{1}\right)(S)$ which we denote by $\mathcal{F}_{\varphi}$. Sending $\varphi$ to $\mathcal{F}_{\varphi}$ defines a fully faithful functor

$$
\mathcal{M}(S) \longrightarrow \mathcal{E} x t\left(E, F_{1}\right)(S)
$$

because for every morphism $\alpha: \mathcal{F}_{\varphi} \rightarrow \mathcal{F}_{\psi}$ in $\mathcal{E} x t\left(E, F_{1}\right)(S)$, its restrictions $\alpha_{U}$ to $U \times_{k} S$ and $\alpha_{V}$ to $V \times_{k} S$ form a morphism $\varphi \rightarrow \psi$ in $\mathcal{M}(S)$ and conversely. 
The functors (2) are compatible with pull-backs along morphisms $f: T \rightarrow S$ of schemes over $A$, so they induce a 1-morphism of stacks over $A$

$$
C^{1}\left(E, F_{1}\right) / C^{0}\left(E, F_{1}\right) \longrightarrow \mathcal{E} x t\left(E, F_{1}\right)
$$

which is in fact an isomorphism because (2) is essentially surjective for affine $S$, since $p^{*} E$ is then projective over $U \times_{k} S$ and over $V \times_{k} S$. The same holds for $E^{\prime}$ instead of $E$, and the 1-isomorphism (3) commutes with $i^{*}$ by construction.

We denote the mapping cone of $i^{*}: C^{\bullet}\left(E, F_{1}\right) \rightarrow C^{\bullet}\left(E^{\prime}, F_{1}\right)$ by $C^{\bullet}\left(F_{2}, F_{1}\right)$; this is a length 2 complex of flat $A$-modules whose cohomology is finitely generated over $A$ by EGA III [13 2.2.1]. Hence there is a quasi-isomorphism

$$
V^{\bullet}=\left[V^{0} \stackrel{\delta^{0}}{\longrightarrow} V^{1} \stackrel{\delta^{1}}{\longrightarrow} V^{2}\right] \stackrel{\sim}{\longrightarrow} C^{\bullet}\left(F_{2}, F_{1}\right)
$$

from a length 2 complex of finitely generated flat $A$-modules $V^{\nu}$, i. e. of vector bundles on $\operatorname{Spec}(A)$; cf. [13, 6.10.5 and 7.7.12.i]. For each $A$-algebra $B$, we have $\mathrm{H}^{\nu}\left(V^{\bullet} \otimes_{A} B\right) \cong \operatorname{Ext}^{\nu}\left(F_{2} \otimes_{A} B, F_{1} \otimes_{A} B\right)$ by construction. If $B$ is a field, then $\operatorname{Ext}^{2}$ vanishes since $C$ is a smooth curve; hence $\delta^{1}: V^{1} \rightarrow V^{2}$ is surjective by Nakayama's lemma, and replacing $V^{1}$ by its kernel if necessary, we may assume $V^{2}=0$. In this situation, it is straightforward to check that the diagram of Picard stacks

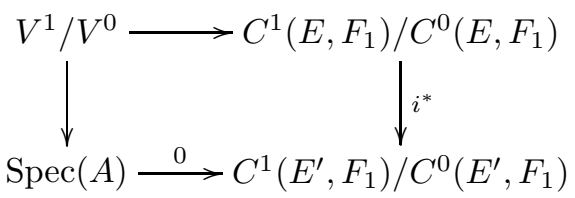

is 1-cartesian as well; here $V^{1} / V^{0}$ is by definition the stack quotient of the total space $\operatorname{Tot}\left(V^{1}\right)$ modulo the action of the algebraic group $V^{0} / A$ given by $\delta^{0}$. Comparing the diagrams (11) and (4) shows that $\mathcal{E} x t\left(F_{2}, F_{1}\right)$ and $V^{1} / V^{0}$ are 1-isomorphic; using the Riemann-Roch formula for $\operatorname{rk}\left(V^{0}\right)-\operatorname{rk}\left(V^{1}\right)$, the lemma follows.

By a vector bundle $\mathcal{V}$ of rank $n$ on a stack $\mathcal{M}$ over $k$, we mean a 1-morphism $\mathcal{V}: \mathcal{M} \rightarrow \mathcal{V} e c t_{n}$. So $\mathcal{V}$ assigns to each object $\mathcal{E}$ of $\mathcal{M}(S)$ a vector bundle $\mathcal{V}(\mathcal{E})$ on the $k$-scheme $S$, in a suitably functorial way; cf. [26. Prop. 13.3.6]. More explicitly, $\mathcal{V}$ is given by a functor $\mathcal{V}(S): \mathcal{M}(S) \rightarrow \mathcal{V}{ }^{2} t_{n}(S)$ for each $k$-scheme $S$, and an isomorphism of functors $f^{*} \circ \mathcal{V}(S) \cong \mathcal{V}(T) \circ f^{*}$ for each $k$-morphism $f: T \rightarrow S$.

Examples 1.11. We fix $r_{1}, r_{2} \geq 1$ and two line bundles $L_{1}, L_{2}$ on $C$.

i) By semicontinuity, there is an open substack $\emptyset \neq \mathcal{U} \subseteq \mathcal{B} u n_{r_{1}, L_{1}} \times \mathcal{B} u n_{r_{2}, L_{2}}$ of vector bundles $E_{1}, E_{2}$ for which $\operatorname{dim} \operatorname{Ext}^{1}\left(E_{1}, E_{2}\right)$ is minimal, say equal to $e$. The vector spaces $\operatorname{Hom}\left(E_{1}, E_{2}\right)$ are the fibers of a vector bundle $\operatorname{Hom}\left(\mathcal{E}_{1}^{\text {univ }}, \mathcal{E}_{2}^{\text {univ }}\right)$ on $\mathcal{U}$ due to the theory of cohomology and base change in EGA III [13, Prop. 7.8.4], using that $\mathcal{U}$ is locally noetherian and reduced. According to Riemann-Roch, its $\operatorname{rank}$ is $\operatorname{rk}\left(\operatorname{Hom}\left(\mathcal{E}_{1}^{\text {univ }}, \mathcal{E}_{2}^{\text {univ }}\right)\right)=(1-g) r_{1} r_{2}+r_{1} \operatorname{deg}\left(L_{2}\right)-r_{2} \operatorname{deg}\left(L_{1}\right)+e$.

ii) We moreover fix a vector bundle $E_{1}$ on $C$ with $\operatorname{rk}\left(E_{1}\right)=r_{1}$ and $\operatorname{det}\left(E_{1}\right) \cong L_{1}$. Similarly, there is an open substack $\emptyset \neq \mathcal{U}_{E_{1}}^{\prime} \subseteq \mathcal{B} u n_{r_{2}, L_{2}}$ where $\operatorname{dim} \operatorname{Ext}^{1}\left(E_{1}, E_{2}\right)$ is minimal, say equal to $e_{E_{1}}$, and on that a vector bundle $\operatorname{Hom}\left(E_{1}, \mathcal{E}_{2}^{\text {univ }}\right)$ with fibers $\operatorname{Hom}\left(E_{1}, E_{2}\right)$. This $\operatorname{Hom}\left(E_{1}, \mathcal{E}_{2}^{\text {univ }}\right)$ is the restriction of $\operatorname{Hom}\left(\mathcal{E}_{1}^{\text {univ }}, \mathcal{E}_{2}^{\text {univ }}\right)$ to the moduli point of $E_{1}$ if the latter is general, more precisely if $e_{E_{1}}=e$.

iii) Arguing as in ii for the other variable, we also obtain for each vector bundle $E_{2}$ on $C$ a vector bundle $\operatorname{Hom}\left(\mathcal{E}_{1}^{\text {univ }}, E_{2}\right)$ on an open substack $\emptyset \neq \mathcal{U}_{E_{2}}^{\prime \prime} \subseteq \mathcal{B} u n_{r_{1}, L_{1}}$. 
As in 21], a key ingredient to our main result will be a variant of Hirschowitz' theorem [17] that the tensor product of two general vector bundles is nonspecial. More precisely, we will use the following:

Proposition 1.12. Given $r_{1}>r_{2} \geq 1$ and two line bundles $L_{1}, L_{2}$ on $C$ with

$$
\operatorname{deg}\left(L_{2}\right) / r_{2}-\operatorname{deg}\left(L_{1}\right) / r_{1}>g-1,
$$

there is a surjective morphism $\varphi: E_{1} \rightarrow E_{2}$ of vector bundles on $C$ with $\operatorname{rk}\left(E_{\nu}\right)=r_{\nu}$ and $\operatorname{det}\left(E_{\nu}\right) \cong L_{\nu}$ such that $\operatorname{Ext}^{1}\left(E_{1}, E_{2}\right)=0$.

Proof. Like [21, Lemma 2.1], this proof is similar to the one given by Russo and Teixidor 35] Thm. 1.2] for Hirschowitz' theorem itself. Since these references don't cover the fixed determinant case that we need, we recall the main arguments here.

Let $\emptyset \neq \mathcal{U} \subseteq \mathcal{B} u n_{r_{1}, L_{1}} \times \mathcal{B} u n_{r_{2}, L_{2}}$ be the open substack of vector bundles $E_{1}$ and $E_{2}$ with $\operatorname{dim} \operatorname{Ext}^{1}\left(E_{1}, E_{2}\right)$ minimal, say equal to $e$. According to generic flatness 14 $\S 6.9]$, there is an open substack $\emptyset \neq \mathcal{M}$ in the total space of the vector bundle $\operatorname{Hom}\left(\mathcal{E}_{1}^{\text {univ }}, \mathcal{E}_{2}^{\text {univ }}\right)$ over $\mathcal{U}$ such that the cokernel of the universal family $\varphi^{\text {univ }}$ of morphisms $\varphi: E_{1} \rightarrow E_{2}$ is flat over $\mathcal{M}$. Then the image of $\varphi^{\text {univ }}$ is is also flat over $\mathcal{M}$, hence a vector bundle over $C \times_{k} \mathcal{M}$, say of rank $r$ and of degree $d$ over every point of $\mathcal{M}$. Since $\mathcal{M}(k) \neq \emptyset$ according to lemma [1.5] it suffices to show $e=0$, $r=r_{2}$ and $d=\operatorname{deg}\left(L_{2}\right)$. Note that $r>0$ by assumption (5) and Riemann-Roch.

By construction, $\mathcal{M}$ is an irreducible smooth algebraic stack over $k$ with

(6) $\operatorname{dim} \mathcal{M}=(g-1)\left(r_{1}^{2}+r_{2}^{2}\right)-2 g+(1-g) r_{1} r_{2}+r_{1} \operatorname{deg}\left(L_{2}\right)-r_{2} \operatorname{deg}\left(L_{1}\right)+e$

due to Riemann-Roch. On the other hand, sending $\varphi: E_{1} \rightarrow E_{2}$ to its kernel, image and cokernel defines a 1-morphism

$$
\mathcal{M} \longrightarrow \mathcal{D} \subseteq \mathcal{B} u n_{r_{1}-r, \operatorname{deg}\left(L_{1}\right)-d} \times \mathcal{B} u n_{r, d} \times \mathcal{C} o h_{r_{2}-r, \operatorname{deg}\left(L_{2}\right)-d}
$$

to the closed substack $\mathcal{D}$ of triples $F_{1}, F, F_{2}$ with $\operatorname{det}\left(F_{\nu}\right) \otimes \operatorname{det}(F) \cong L_{\nu}$ for $\nu=1,2$. Now $\mathcal{D}$ is irreducible of codimension $2 g$ (since $r_{1}-r>0$ and $r>0$ ), so

$$
\operatorname{dim} \mathcal{D}=(g-1)\left[\left(r_{1}-r\right)^{2}+r^{2}+\left(r_{2}-r\right)^{2}\right]-2 g .
$$

The points $\varphi: E_{1} \rightarrow E_{2}$ in $\mathcal{M}$ with given image in $\mathcal{D}$ correspond to extensions

$$
0 \rightarrow \operatorname{ker}(\varphi) \rightarrow E_{1} \rightarrow \operatorname{im}(\varphi) \rightarrow 0 \quad \text { and } \quad 0 \rightarrow \operatorname{im}(\varphi) \rightarrow E_{2} \rightarrow \operatorname{coker}(\varphi) \rightarrow 0
$$

that satisfy the open conditions ' $E_{2}$ torsionfree' and ' $\operatorname{dim} \operatorname{Ext}^{1}\left(E_{1}, E_{2}\right) \leq e$ '; using lemma 1.10 this means that $\mathcal{M}$ is smooth over $\mathcal{D}$ of relative dimension

$$
\begin{aligned}
\operatorname{dim} \mathcal{M}-\operatorname{dim} \mathcal{D}= & (g-1)\left(r_{1}-r\right) r+\left(r_{1}-r\right) d-r\left(\operatorname{deg} L_{1}-d\right) \\
+ & (g-1) r\left(r_{2}-r\right)+r\left(\operatorname{deg} L_{2}-d\right)-\left(r_{2}-r\right) d
\end{aligned}
$$

Combining the dimension formulas (6), (7) and (8) yields

$$
\left(r_{1}-r\right)\left(\operatorname{deg} L_{2}-d\right)-\left(r_{2}-r\right)\left(\operatorname{deg} L_{1}-d\right)+e=(g-1)\left(r_{1}-r\right)\left(r_{2}-r\right) .
$$

Now suppose $r<r_{2}$. The image of $\mathcal{M}$ in $\mathcal{B} u n_{r_{1}, L_{1}} \times \mathcal{B} u n_{r_{2}, L_{2}}$ is open and dense, so it contains a pair $E_{1}, E_{2}$ of stable vector bundles on $C$. Consequently, the slopes $\mu_{\nu}:=\left(\operatorname{deg} L_{\nu}-d\right) /\left(r_{\nu}-r\right)$ of $\operatorname{ker}(\varphi)$ and $\operatorname{coker}(\varphi)$ satisfy $\mu_{1}<\operatorname{deg}\left(L_{1}\right) / r_{1}$ and $\operatorname{deg}\left(L_{2}\right) / r_{2}<\mu_{2}$. On the other hand, $\mu_{2}-\mu_{1} \leq g-1$ by equation (9). This leads to a contradiction with our assumption (5), thereby proving $r=r_{2}$.

Now equation (9) reads $\left(r_{1}-r\right)\left(\operatorname{deg} L_{2}-d\right)+e=0$. But $d \leq \operatorname{deg}\left(L_{2}\right)$ because otherwise $\mathcal{C}_{o} h_{r_{2}-r, \operatorname{deg}\left(L_{2}\right)-d}$ would be empty, $r_{1}-r \geq r_{1}-r_{2}>0$ by hypothesis and of course also $e \geq 0$; thus we conclude $\operatorname{deg}\left(L_{2}\right)=d$ and $e=0$. 


\section{2. $\mathbb{G}_{m}$-STACKS AND $\mathbb{G}_{m}$-GERBES}

Definition 2.1. A $\mathbb{G}_{m}$-stack $\mathcal{M}=(\mathcal{M}, \iota)$ over $k$ consists of an algebraic stack $\mathcal{M}$ over $k$ together with a group homomorphism $\iota(\mathcal{E}): \Gamma\left(S, \mathcal{O}_{S}^{*}\right) \rightarrow \operatorname{Aut}_{\mathcal{M}(S)}(\mathcal{E})$ for each $k$-scheme $S$ and each object $\mathcal{E}$ of the groupoid $\mathcal{M}(S)$ such that the diagrams
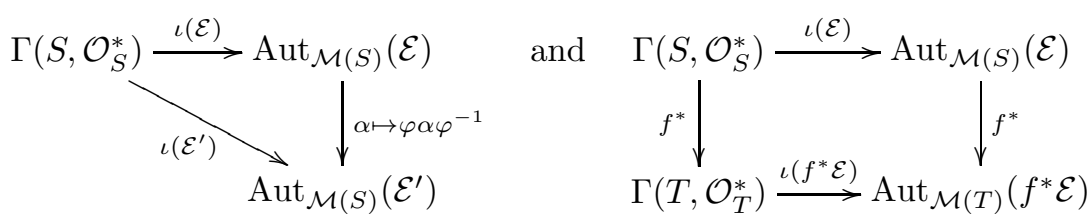

commute for each morphism $\varphi: \mathcal{E} \rightarrow \mathcal{E}^{\prime}$ in $\mathcal{M}(S)$ and each $k$-morphism $f: T \rightarrow S$.

Examples 2.2. The moduli stacks of vector bundles $\mathcal{B} u n_{r, d}$ and $\mathcal{B} u n_{r, L}$ are in a canonical way $\mathbb{G}_{m}$-stacks: The multiplication by scalars defines a canonical homomorphism $\iota(\mathcal{E}): \Gamma\left(S, \mathcal{O}_{S}^{*}\right) \rightarrow \operatorname{Aut}(\mathcal{E})$ for each $k$-scheme $S$ and each vector bundle $\mathcal{E}$ on $C \times_{k} S$; the functoriality conditions above are obviously satisfied.

Definition 2.3. A $\mathbb{G}_{m}$-stack $(\mathcal{M}, \iota)$ is a $\mathbb{G}_{m}$-gerbe if $\iota(\mathcal{E}): \Gamma\left(S, \mathcal{O}_{S}^{*}\right) \rightarrow \operatorname{Aut}_{\mathcal{M}(S)}(\mathcal{E})$ is an isomorphism for every $k$-scheme $S$ and every object $\mathcal{E}$ of $\mathcal{M}(S)$.

Examples 2.4. The open substacks $\mathcal{B} u n_{r, d}^{\text {stab }} \subseteq \mathcal{B} u n_{r, d}$ and $\mathcal{B} u n_{r, L}^{\text {stab }} \subseteq \mathcal{B} u n_{r, L}$ of geometrically stable vector bundles $E$ on $C$ are $\mathbb{G}_{m}$-gerbes.

Proof. Geometrically stable vector bundles $E$ in $C$ are known to be simple, i. e. $\operatorname{End}(E)=k \cdot \mathrm{id}_{E}$. If $S$ is a $k$-scheme and $\mathcal{E}$ is a vector bundle on $C \times_{k} S$ whose restriction to each point of $S$ is simple, then the theory of cohomology and base change in EGA III 13] shows $\operatorname{End}(\mathcal{E})=\Gamma\left(S, \mathcal{O}_{S}\right) \cdot \mathrm{id}_{\mathcal{E}}$. (Strictly speaking, 13] applies only if $S$ is locally noetherian, but standard limit arguments allow us to assume that.) Hence $\iota(\mathcal{E}): \Gamma\left(S, \mathcal{O}_{S}^{*}\right) \rightarrow \operatorname{Aut}(\mathcal{E})$ is indeed an isomorphism.

Example 2.5. If $\mathfrak{M}$ is an algebraic space locally of finite type over $k$, then the stack $\mathcal{M}:=\mathfrak{M} \times{ }_{k} \mathrm{~B} \mathbb{G}_{m}$ is a $\mathbb{G}_{m}$-gerbe over $k$ in an obvious way.

Definition 2.6. Let $(\mathcal{M}, \iota)$ and $\left(\mathcal{M}^{\prime}, \iota^{\prime}\right)$ be $\mathbb{G}_{m}$-stacks over $k$. A 1-morphism $\Phi: \mathcal{M} \rightarrow \mathcal{M}^{\prime}$ has weight $w \in \mathbb{Z}$ if the diagram

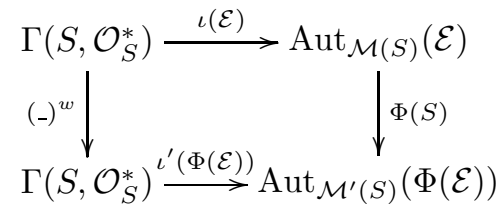

commutes for every $k$-scheme $S$ and every object $\mathcal{E}$ of the groupoid $\mathcal{M}(S)$.

Example 2.7. There is a canonical 1-isomorphism $\mathcal{B} u n_{r, L} \rightarrow \mathcal{B} u n_{r, L^{\text {dual }}}$ of stacks over $k$ which has weight -1 : It sends a vector bundle $\mathcal{E}$ on $C \times_{k} S$ to $\mathcal{E}^{\text {dual }}$ and an isomorphism $\varphi: \mathcal{E}_{1} \rightarrow \mathcal{E}_{2}$ of vector bundles on $C \times{ }_{k} S$ to $\left(\varphi^{-1}\right)^{\text {dual }}: \mathcal{E}_{1}^{\text {dual }} \rightarrow \mathcal{E}_{2}^{\text {dual }}$.

Lemma 2.8. Let $(\mathcal{M}, \iota)$ and $\left(\mathcal{M}^{\prime}, \iota^{\prime}\right)$ be $\mathbb{G}_{m}$-gerbes over $k$. If $\mathcal{M}$ is connected, then every 1-isomorphism $\Phi: \mathcal{M} \rightarrow \mathcal{M}^{\prime}$ has weight 1 or has weight -1 .

Proof. Let $S$ be a $k$-scheme, and let $\mathcal{E}$ be an object of $\mathcal{M}(S)$. For each morphism of $k$-schemes $f: T \rightarrow S$, we consider the group isomorphism

$$
\Gamma\left(T, \mathcal{O}_{T}^{*}\right) \cong \operatorname{Aut}_{\mathcal{M}(T)}\left(f^{*} \mathcal{E}\right) \stackrel{\Phi(T)}{\longrightarrow} \operatorname{Aut}_{\mathcal{M}^{\prime}(T)}\left(\Phi\left(f^{*} \mathcal{E}\right)\right) \cong \Gamma\left(T, \mathcal{O}_{T}\right) .
$$


As $T$ varies, these form an automorphism of the group scheme $\mathbb{G}_{m}$ over $S$. Hence $S$ is a disjoint union of open subschemes $S_{+}$and $S_{-}$where this automorphism is the identity and $\lambda \mapsto \lambda^{-1}$, respectively. This shows that $\mathcal{M}$ is the disjoint union of open substacks $\mathcal{M}_{+}$and $\mathcal{M}_{-}$where $\Phi$ has weight 1 and weight -1 , respectively; since $\mathcal{M}$ is connected, one of these is empty.

Recall that the coarse space $\mathfrak{M}$ associated to an algebraic stack $\mathcal{M}$ over $k$ is the fppf-sheafification of the functor (Schemes $/ k$ ) op $^{\text {op }} \rightarrow$ Sets that sends each $k$-scheme $S$ to the set of isomorphism classes in the groupoid $\mathcal{M}(S)$. We say that $\mathfrak{M}$ is a scheme or an algebraic space if this sheafified functor is representable by such.

Examples 2.9. The coarse spaces associated to the $\mathbb{G}_{m}$-gerbes $\mathcal{B} u n_{r, d}^{\text {stab }}$ and $\mathcal{B} u n_{r, L}^{\text {stab }}$ are the usual quasiprojective coarse moduli schemes $\mathfrak{B u n}_{r, d}$ and $\mathfrak{B u n}_{r, L}$ of stable vector bundles $E$ on $C$. We denote by $\mathfrak{B u n}_{r, d} \subseteq \overline{\mathfrak{B u n}}_{r, d}$ and by $\mathfrak{B u n}_{r, L} \subseteq \overline{\mathfrak{B u n}}_{r, L}$ the natural compactifications given by semistable vector bundles.

Remark 2.10. Each $\mathbb{G}_{m}$-gerbe $\mathcal{M}$ in the sense above is a gerbe with band $\mathbb{G}_{m}$ over its coarse space $\mathfrak{M}$ in the sense of [12, Déf. IV.2.2.2]; cf. also [26, Rem. 3.19]. This justifies the terminology ' $\mathbb{G}_{m}$-gerbe'.

Lemma 2.11. Let $\mathfrak{M}$ be the coarse space associated to a $\mathbb{G}_{m}$-gerbe $\mathcal{M}$ over $k$.

i) $\mathfrak{M}$ is an algebraic space locally of finite type over $k$.

ii) The canonical 1-morphism $\pi: \mathcal{M} \rightarrow \mathfrak{M}$ is faithfully flat of finite presentation.

iii) The induced map of points $|\pi|:|\mathcal{M}| \rightarrow|\mathfrak{M}|$ is a homeomorphism for the Zariski topologies defined by [25] Def. II.6.9] and [26, 5.5].

Proof. Due to [26. Cor. 10.8], $\mathfrak{M}$ is an algebraic space, and $\pi$ is faithfully flat and locally of finite presentation. The induced map $|\pi|:|\mathcal{M}| \rightarrow|\mathfrak{M}|$ is automatically continuous, and it is bijective by the definition [26. Déf. 5.2] of point; $|\pi|$ is also open since $\pi$ is flat 26. Prop. 5.6]. This proves iii; in particular, $\pi$ is quasicompact, which completes the proof of ii. Using [14, 2.7.1 and 17.7.5], ii implies that $\mathfrak{M}$ is locally of finite type over $k$ because $\mathcal{M}$ is so, by our convention on stacks.

Remarks 2.12. i) The coarse space $\pi: \mathcal{M} \rightarrow \mathfrak{M}$ associated to a $\mathbb{G}_{m}$-gerbe $\mathcal{M}$ over $k$ is by definition universal for morphisms from $\mathcal{M}$ to algebraic spaces over $k$; in particular, $\mathfrak{M}$ is reduced if $\mathcal{M}$ is. Part iii of the above lemma 2.11]implies that $\mathfrak{M}$ is irreducible if $\mathcal{M}$ is. Hence $\mathfrak{M}$ is integral if $\mathcal{M}$ is.

ii) If irreducible $\mathbb{G}_{m}$-gerbes $\mathcal{M}$ and $\mathcal{M}^{\prime}$ over $k$ are birational, then their coarse spaces $\mathfrak{M}$ and $\mathfrak{M}^{\prime}$ are birational as well, due to the above lemma 2.11 iii again.

A $\mathbb{G}_{m}$-gerbe $\mathcal{M}$ is called neutral if the canonical 1-morphism to its coarse space $\pi: \mathcal{M} \rightarrow \mathfrak{M}$ admits a section $s: \mathfrak{M} \rightarrow \mathcal{M}$. If this is the case, then there is a 1-isomorphism $\mathcal{M} \cong \mathfrak{M} \times \mathrm{B} \mathbb{G}_{m}$ of weight 1 according to [26, Lemme 3.21].

Remark 2.13. Lemma 2.11 ii above implies that every $\mathbb{G}_{m}$-gerbe $\mathcal{M}$ over $k$ is locally neutral for the fppf-topology on its coarse space $\mathfrak{M}$.

Definition 2.14. Let $\pi: \mathcal{M} \rightarrow \mathfrak{M}$ be the coarse space associated to a $\mathbb{G}_{m}$-gerbe $\mathcal{M}$ over $k$, and let $p: S \rightarrow \mathfrak{M}$ be a scheme over $\mathfrak{M}$. A Poincaré family on $S$ is an object $\mathcal{E}$ of $\mathcal{M}(S)$ whose classifying morphism $c_{\mathcal{E}}: S \rightarrow \mathcal{M}$ satisfies $p=\pi \circ c_{\mathcal{E}}$.

Let $S \rightarrow \mathfrak{M} \leftarrow \mathcal{M}$ still be a scheme over the coarse space $\mathfrak{M}$ of a $\mathbb{G}_{m}$-gerbe $\mathcal{M}$. Then $\mathcal{S}:=\mathcal{M} \times_{\mathfrak{M}} S$ is a $\mathbb{G}_{m}$-gerbe with coarse space $S$; this $\mathbb{G}_{m}$-gerbe is neutral if and only if there is a Poincaré family $\mathcal{E}$ on $S$. 


\section{Vector Bundles on $\mathbb{G}_{m}$-Gerbes}

Definition 3.1. Let $(\mathcal{M}, \iota)$ be a $\mathbb{G}_{m}$-stack over $k$. A vector bundle $\mathcal{V}$ on $\mathcal{M}$ has weight $w \in \mathbb{Z}$ if the diagram

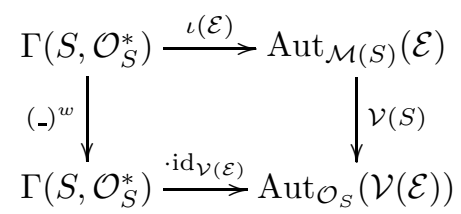

commutes for every $k$-scheme $S$ and every object $\mathcal{E}$ of the groupoid $\mathcal{M}(S)$.

Example 3.2. The trivial vector bundle $\mathcal{O}^{n}$ on any $\mathbb{G}_{m}$-stack $\mathcal{M}$ has weight 0.

Examples 3.3. Given a point $P \in C(k)$, we denote by $\mathcal{E}_{P}^{\text {univ }}$ the restriction of the universal vector bundle $\mathcal{E}^{\text {univ }}$ on $C \times \mathcal{B} u n_{r, L}$ to $\{P\} \times \mathcal{B} u n_{r, L} \cong \mathcal{B} u n_{r, L}$.

i) $\mathcal{E}_{P}^{\text {univ }}$ is a vector bundle of weight 1 on $\mathcal{B} u n_{r, L}$.

ii) $\left(\mathcal{E}_{P}^{\text {univ }}\right)^{\text {dual }}$ is a vector bundle of weight -1 on $\mathcal{B} u n_{r, L}$.

Examples 3.4. We fix $r \in \mathbb{N}$, a line bundle $L$ and a vector bundle $F$ on $C$. Using the notation of the examples 1.11 ii and iii, we have:

i) The vector bundle $\operatorname{Hom}\left(F, \mathcal{E}^{\text {univ }}\right)$ on $\mathcal{U}_{F}^{\prime} \subseteq \mathcal{B}$ un $n_{r, L}$ has weight 1 .

ii) The vector bundle $\operatorname{Hom}\left(\mathcal{E}^{\text {univ }}, F\right)$ on $\mathcal{U}_{F}^{\prime \prime} \subseteq \mathcal{B}$ un $n_{r, L}$ has weight -1 .

Example 3.5. The formalism of determinant line bundles 24 yields a line bundle $\mathcal{L}_{\text {det }}$ on $\mathcal{B} u n_{r, L}$ whose fibre over any point $[E]$ is $\operatorname{det} \mathrm{H}^{0}(E) \otimes \operatorname{det}^{-1} \mathrm{H}^{1}(E)$. This $\mathcal{L}_{\text {det }}$ is a line bundle of weight $r(1-g)+\operatorname{deg}(L)$ on $\mathcal{B} u n_{r, L}$ by Riemann-Roch.

We recall that vector bundles $\mathcal{V}$ on a fixed algebraic stack $\mathcal{M}$ over $k$ form a category; a morphism $\mathcal{V} \rightarrow \mathcal{W}$ of such vector bundles can be described by a morphism $\mathcal{V}(\mathcal{E}) \rightarrow \mathcal{W}(\mathcal{E})$ of vector bundles on the $k$-scheme $S$ for every object $\mathcal{E}$ of $\mathcal{M}(S)$.

Lemma 3.6. Let $\pi: \mathcal{M} \rightarrow \mathfrak{M}$ be the coarse space associated to a $\mathbb{G}_{m}$-gerbe $\mathcal{M}$ over $k$. Sending $V$ to $\mathcal{V}:=\pi^{*}(V)$ defines an equivalence between the category of vector bundles $V$ on $\mathfrak{M}$ and the category of weight 0 vector bundles $\mathcal{V}$ on $\mathcal{M}$.

Proof. We claim that the inverse equivalence is given by the functor $\pi_{*}$ from quasi-

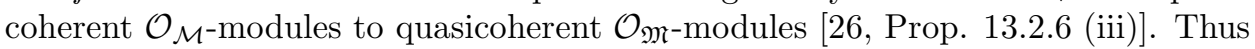
we have to show that $\pi_{*}$ sends weight 0 vector bundles $\mathcal{V}$ on $\mathcal{M}$ to vector bundles $V$ on $\mathfrak{M}$ and that the two adjunction morphisms $\pi^{*} \pi_{*} \mathcal{V} \rightarrow \mathcal{V}$ and $V \rightarrow \pi_{*} \pi^{*} V$ are isomorphisms. All this can be verified locally in the fppf-topology on $\mathfrak{M}$, so we may assume that the $\mathbb{G}_{m}$-gerbe $\mathcal{M}$ is neutral; this special case is easy to check.

Example 3.7. If $\operatorname{deg}(L)=r(g-1)$, then the determinant line bundle $\mathcal{L}_{\text {det }}$ on $\mathcal{B} u n_{r, L}$ has weight 0 ; thus its restriction to $\mathcal{B} u n_{r, L}^{\text {stab }}$ descends to $\mathfrak{B u n}_{r, L}$ by lemma 3.6 In fact, $\mathcal{L}_{\text {det }}$ descends to a line bundle $\mathfrak{L}_{\text {det }}$ on $\overline{\mathfrak{B u n}}_{r, L}$ by [23, Prop. 4.2].

Corollary 3.8. Let $\mathcal{V}$ be a vector bundle of weight 0 on an irreducible $\mathbb{G}_{m}$-gerbe $\mathcal{M}$ over $k$. Then $\mathcal{V}$ is trivial on some open substack $\emptyset \neq \mathcal{U} \subseteq \mathcal{M}$.

Proof. We have $\mathcal{V} \cong \pi^{*}(V)$ for a vector bundle $V$ on $\mathfrak{M}$. [25, Prop. II.6.7] states that some open subspace $\emptyset \neq \mathfrak{U} \subseteq \mathfrak{M}$ is a scheme; hence $V$ is trivial on some open subscheme $\emptyset \neq U \subseteq \mathfrak{U}$, and $\mathcal{V}$ is trivial on $\mathcal{U}:=\pi^{-1}(U)$. 
Lemma 3.9. Let $\mathcal{M}$ be a $\mathbb{G}_{m}$-gerbe over $k$ with coarse space $\pi: \mathcal{M} \rightarrow \mathfrak{M}$. This $\mathbb{G}_{m}$-gerbe is neutral if and only if there is a line bundle $\mathcal{L}$ of weight 1 on $\mathcal{M}$.

Proof. If $\mathcal{M}$ is neutral, say $\mathcal{M}=\mathfrak{M} \times \mathrm{B} \mathbb{G}_{m}$, then pulling back the canonical line bundle $L^{\text {univ }}$ of weight 1 on $B \mathbb{G}_{m}$ yields a line bundle of weight 1 on $\mathcal{M}$.

Conversely, suppose that there is a line bundle $\mathcal{L}$ of weight 1 on $\mathcal{M}$, and let $p: \operatorname{Tot}(\mathcal{L})^{*} \rightarrow \mathcal{M}$ be the complement of the zero section in its total space. We claim that $p$ is a section for $\pi$, i. e. that $\pi \circ p: \operatorname{Tot}(\mathcal{L})^{*} \rightarrow \mathfrak{M}$ is a 1-isomorphism. This can be checked locally in the fppf-topology on $\mathfrak{M}$, so we may assume $\mathcal{M}=\mathfrak{M} \times \mathrm{B} \mathbb{G}_{m}$. Now $\mathcal{L}$ and the pull-back of $L^{\text {univ }}$ differ by tensoring with a weight 0 line bundle, so they are isomorphic locally in the fppf-topology on $\mathfrak{M}$ by lemma 3.6] hence we may even assume that $\mathcal{L}$ is the pull-back of $L^{\text {univ }}$. This special case is easy to check.

Lemma 3.10. Let $\mathcal{M}$ be an integral $\mathbb{G}_{m}$-gerbe over $k$ with coarse space $\mathcal{M} \stackrel{\pi}{\rightarrow} \mathfrak{M}$.

i) There is a vector bundle $\mathcal{V}_{1}$ of weight 1 on some open substack $\emptyset \neq \mathcal{U}_{1} \subseteq \mathcal{M}$.

ii) The generic fibre of the algebra bundle End $\left(\mathcal{V}_{1}\right)$ on $\mathcal{U}_{1}$ descends to a central simple algebra $A$ over the function field $k(\mathfrak{M})$.

iii) The Brauer class $\psi_{\mathcal{M}}:=[A] \in \operatorname{Br}(k(\mathfrak{M}))$ of the central simple algebra $A$ in ii does not depend on the choice of $\mathcal{U}_{1}$ and $\mathcal{V}_{1}$ made in $i$.

iv) Any two vector bundles $\mathcal{V}, \mathcal{V}^{\prime}$ of the same rank $n$ and weight $w$ on open substacks $\emptyset \neq \mathcal{U}, \mathcal{U}^{\prime} \subseteq \mathcal{M}$ are isomorphic on some open substack $\emptyset \neq \mathcal{U}^{\prime \prime} \subseteq \mathcal{U} \cap \mathcal{U}^{\prime}$.

$v)$ There is a vector bundle of given rank $n$ and given weight $w$ on some open substack $\emptyset \neq \mathcal{U} \subseteq \mathcal{M}$ if and only if the index of $w \cdot \psi_{\mathcal{M}} \in \operatorname{Br}(k(\mathfrak{M}))$ divides $n$.

vi) There is a Poincaré family $\mathcal{E}$ on some integral, generically finite scheme $S$ of given degree $n$ over $\mathfrak{M}$ if and only if the index of $\psi_{\mathcal{M}}$ itself divides $n$.

Proof. Replacing the base field $k$ by the function field $k(\mathfrak{M})$ and $\mathcal{M}$ by the $\mathbb{G}_{m^{-}}$ gerbe $\mathcal{M} \times \mathfrak{M} \operatorname{Spec}(k(\mathfrak{M}))$ over it, we may assume $\mathfrak{M}=\operatorname{Spec}(k)$; then the only open substack $\emptyset \neq \mathcal{U} \subseteq \mathcal{M}$ is $\mathcal{U}=\mathcal{M}$ itself.

i) Due to Hilbert's Nullstellensatz, there is a finite field extension $k_{1} \supseteq k$ with $\mathcal{M}\left(k_{1}\right) \neq \emptyset$. Then the $\mathbb{G}_{m}$-gerbe $\mathcal{M}_{1}:=\mathcal{M} \times_{k} \operatorname{Spec}\left(k_{1}\right)$ is neutral, so there is a line bundle $\mathcal{L}_{1}$ of weight 1 on $\mathcal{M}_{1}$ by lemma 3.9. Since the first projection pr: $\mathcal{M}_{1} \rightarrow \mathcal{M}$ is finite, flat and has weight 1 , we get a vector bundle $\operatorname{pr}_{*} \mathcal{L}_{1}$ of weight 1 on $\mathcal{M}$.

ii) Let $\mathcal{V}_{1}$ be a vector bundle of weight 1 and $\operatorname{rank} n_{1}$ on $\mathcal{U}_{1}=\mathcal{M}$. The vector bundle $\operatorname{End}\left(\mathcal{V}_{1}\right)$ of weight 0 on $\mathcal{M}$ and its multiplication descend to an algebra $A$ of dimension $n_{1}^{2}$ over $k$ by lemma 3.6] With $k_{1}$, pr: $\mathcal{M}_{1} \rightarrow \mathcal{M}$ and $\mathcal{L}_{1}$ as in i,

$$
A \otimes_{k} k_{1} \cong \operatorname{End}\left(\operatorname{pr}^{*} \mathcal{V}_{1}\right) \cong \operatorname{End}\left(\operatorname{pr}^{*} \mathcal{V}_{1} \otimes \mathcal{L}_{1}^{\text {dual }}\right)
$$

is a full matrix algebra over $k_{1}$ because the weight 0 vector bundle $\operatorname{pr}^{*} \mathcal{V}_{1} \otimes \mathcal{L}_{1}^{\text {dual }}$ on $\mathcal{M}_{1}$ is trivial by corollary 3.8 this shows that $A$ is central simple over $k$.

iii $-\mathrm{v}$ ) If $\mathcal{V}$ is a vector bundle of rank $n$ and weight $w$ on $\mathcal{M}$, then the weight 0 vector bundle $\operatorname{Hom}\left(\mathcal{V}_{1}^{\otimes w}, \mathcal{V}\right)$ on $\mathcal{M}$ and the right action of $\operatorname{End}\left(\mathcal{V}_{1}\right)^{\otimes w}$ on it descend to a right module $M$ under the $k$-algebra $A^{\otimes w}$ with $\operatorname{dim}_{k}(M)=n_{1}^{w} \cdot n$ by lemma 3.6. This defines a Morita equivalence between vector bundles $\mathcal{V}$ of weight $w$ on $\mathcal{M}$ and finitely generated right $A^{\otimes w}$-modules $M$; its inverse sends $M$ to $\mathcal{V}:=\pi^{*} M \otimes \mathcal{V}_{1}^{\otimes w}$ where the tensor product is taken over $\pi^{*} A^{\otimes w} \cong \operatorname{End}\left(\mathcal{V}_{1}^{\otimes w}\right)$.

In particular, the category of finitely generated right $A$-modules is independent of $\mathcal{V}_{1}$ (up to equivalence); this implies iii. According to the theory of central simple algebras, a right $A^{\otimes w}$-module $M$ is determined up to isomorphism by $\operatorname{dim}_{k}(M)$, 
and there is one with $\operatorname{dim}_{k}(M)=n_{1}^{w} \cdot n$ if and only if the index of $\left[A^{\otimes w}\right]$ divides $n$; this proves iv and $\mathrm{v}$.

vi) Here $S=\operatorname{Spec}(K)$ for a finite field extension $K \supseteq k$. There is a Poincaré family $\mathcal{E}$ on $S=\operatorname{Spec}(K)$ if and only if the $\mathbb{G}_{m}$-gerbe $\mathcal{M}_{K}:=\mathcal{M} \times_{k} \operatorname{Spec}(K)$ is neutral, i. e. if and only if its Brauer class $\psi_{\mathcal{M}_{K}} \in \operatorname{Br}(K)$ vanishes; the latter holds if and only if $K$ is a splitting field for $\psi_{\mathcal{M}}$, because $\psi_{\mathcal{M}_{K}}=\psi_{\mathcal{M}} \otimes_{k} K$ in general. By the theory of central simple algebras again, $\psi_{\mathcal{M}} \in \operatorname{Br}(k)$ has a splitting field of given degree $n$ over $k$ if and only if its index divides $n$.

Remark 3.11. If $\mathcal{M}=\mathcal{B u n}_{r, d}$, then $\psi_{\mathcal{M}}$ is precisely the Brauer class $\psi_{r, d}$ used by King and Schofield [21, Def. 3.3]. The above lemma is based on their arguments.

Remark 3.12. Much more information about vector bundles on $\mathbb{G}_{m}$-gerbes, in particular about their moduli spaces, can be found in M. Lieblich's work [28, 29].

Proposition 3.13. Suppose that there is a line bundle $\mathcal{L}$ of weight $w \in \mathbb{Z}$ on an open substack $\emptyset \neq \mathcal{U} \subseteq \mathcal{B}$ un $_{r, L}$ with $\operatorname{deg}(L)=0$. Then $r$ divides $w$.

Proof. Considering $\mathcal{L} \otimes_{k} \bar{k}$ instead of $\mathcal{L}$, we may assume that $k$ is algebraically closed. Then $L$ is the $r$-th tensor power of a line bundle on $C$, tensoring with which defines an isomorphism $\mathcal{B} u n_{r, \mathcal{O}} \cong \mathcal{B} u n_{r, L}$. Hence we may also assume $L=\mathcal{O}$.

We denote by $\left[\mathcal{O}^{r}\right]$ the point on $\mathcal{B} u n_{r, \mathcal{O}}$ corresponding to the trivial bundle $\mathcal{O}^{r}$ on $C$. Shrinking $\mathcal{U}$ if necessary, we may suppose that $\mathcal{U}$ is quasicompact; then we can extend $\mathcal{L}$ to a neighbourhood of the point $\left[\mathcal{O}^{r}\right]$ as follows:

Choose a quasicompact open substack $\mathcal{U}^{\prime}$ of $\mathcal{B} u n_{r, \mathcal{O}}$ that contains $\mathcal{U}$ and $\left[\mathcal{O}^{r}\right]$. [26. Cor. 15.5] allows us to extend $\mathcal{L}$ to a coherent sheaf on $\mathcal{U}^{\prime}$, more precisely to a coherent subsheaf $\mathcal{F} \subseteq j_{*} \mathcal{L}$ where $j: \mathcal{U} \hookrightarrow \mathcal{U}^{\prime}$ is the open embedding. Then the double dual $\mathcal{L}^{\prime}:=\mathcal{F}^{* *}$ is a reflexive coherent sheaf of rank one on the smooth stack $\mathcal{U}^{\prime}$ and hence a line bundle, cf. 6] Chap. VII, §4.2].

Now $\mathcal{L}^{\prime}$ is a line bundle of weight $w$ because its restriction $\mathcal{L}$ to $\mathcal{U}$ is so. In particular, the scalars $\mathbb{G}_{m} \subseteq \mathrm{GL}_{r}=\operatorname{Aut}\left(\mathcal{O}^{r}\right)$ act with weight $w$ on the fibre of $\mathcal{L}^{\prime}$ over $\left[\mathcal{O}^{r}\right]$. But any one-dimensional representation of $\mathrm{GL}_{r}$ factors through the determinant, so its weight $w$ is always a multiple of $r$.

Corollary 3.14. We fix a line bundle $L$ of degree 0 on $C, r, n \geq 1$ and $w \in \mathbb{Z}$.

i) There is a vector bundle $\mathcal{V}$ of rank $n$ and weight $w$ on some open substack $\emptyset \neq \mathcal{U} \subseteq \mathcal{B u n}_{r, L}$ if and only if $r$ divides $w \cdot n$.

ii) There is a Poincaré family $\mathcal{E}$ on some integral, generically finite scheme $S$ of degree $n$ over $\mathfrak{B u n}_{r, L}$ if and only if $r$ divides $n$.

Proof. If $\mathcal{V}$ is a vector bundle of rank $n$ and weight $w$ on $\mathcal{U}$, then $\mathcal{L}:=\operatorname{det}(\mathcal{V})$ is a line bundle of weight $w \cdot n$; the 'only if' in i thus follows from the previous proposition. According to lemma $3.10 \mathrm{v}$, this means that $r$ divides $w$ times the index of the Brauer class $w \cdot \psi_{\mathcal{B u n}_{r, L}} \in \operatorname{Br}\left(k\left(\mathfrak{B u n} \mathfrak{n}_{r, L}\right)\right)$.

On the other hand, the vector bundle $\mathcal{E}_{P}^{\text {univ }}$ of rank $r$ and weight 1 on $\mathcal{B} u n_{r, L}$ shows that the index of $\psi_{\mathcal{B} u n_{r, L}}$ divides $r$. Due to [2, 5.4], this implies that the index of $w \cdot \psi_{\mathcal{B u n}_{r, L}}$ divides $r / w$ if $w$ divides $r$; together with the previous paragraph, this shows that the index of $w \cdot \psi_{\mathcal{B} u n_{r, L}}$ equals $r / w$ if $w$ divides $r$.

For general $w$, the Brauer classes $\operatorname{hcf}(r, w) \cdot \psi_{\mathcal{B u n}_{r, L}}$ and $w \cdot \psi_{\mathcal{B} u n_{r, L}}$ have the same splitting fields because $w / \operatorname{hcf}(r, w)$ is invertible modulo $r$; hence the index $r / h c f(r, w)$ of the former is also the index of the latter. Using this, i and ii follow from lemma $3.10 \mathrm{v}$ and vi. 


\section{Grassmannian Bundles}

Given a vector bundle $\mathcal{V}$ on a stack $\mathcal{M}$ over $k$, we denote by $\operatorname{Gr}_{j}(\mathcal{V}) \rightarrow \mathcal{M}$ the associated Grassmannian bundle of $j$-dimensional linear subspaces for $j \leq \operatorname{rk}(\mathcal{V})$. Recall that this is a smooth representable 1-morphism of stacks over $k$ whose fibre over the point of $\mathcal{M}$ corresponding to an object $E$ of $\mathcal{M}(k)$ is the usual Grassmannian scheme $\operatorname{Gr}_{j}(\mathcal{V}(E))$.

If $(\mathcal{M}, \iota)$ is a $\mathbb{G}_{m}$-stack over $k$ and $\mathcal{V}$ has some weight $w \in \mathbb{Z}$, then $\operatorname{Gr}_{j}(\mathcal{V})$ becomes a $\mathbb{G}_{m}$-stack over $k$ as well, because all linear subspaces of $\mathcal{V}(E)$ are invariant under the $\mathbb{G}_{m}$-action given by $\iota$. If $\mathcal{M}$ is even a $\mathbb{G}_{m}$-gerbe over $k$, then $\operatorname{Gr}_{j}(\mathcal{V})$ also is.

Remark 4.1. There is a canonical 1-isomorphism $\operatorname{Gr}_{j}(\mathcal{V}) \stackrel{\sim}{\longrightarrow} \operatorname{Gr}_{\operatorname{rk}(\mathcal{V})-j}\left(\mathcal{V}^{\text {dual }}\right)$ that sends each linear subspace of $\mathcal{V}(E)$ to its orthogonal complement in $\mathcal{V}(E)^{\text {dual }}$.

Examples 4.2. We denote by $\mathcal{P a r}_{r, L}^{m / P}$ the moduli stack of rank $r$ vector bundles $E$ on $C$ with $\operatorname{det}(E) \cong L$, endowed with a quasiparabolic structure of multiplicity $m$ over the point $P \in C(k)$ in the sense of 30 . We recall that such a quasiparabolic structure can be given by a coherent subsheaf $E^{\prime} \subseteq E$ for which $E / E^{\prime}$ is isomorphic to the skyscraper sheaf $\mathcal{O}_{P}^{m}$.

i) $\operatorname{Par}_{r, L}^{m / P}$ is canonically 1-isomorphic to $\operatorname{Gr}_{m}\left(\left(\mathcal{E}_{P}^{\text {univ }}\right)^{\text {dual }}\right)$ over $\mathcal{B} u n_{r, L}$.

ii) $\operatorname{Par}_{r, L}^{m / P}$ is also 1-isomorphic to $\operatorname{Gr}_{m}\left(\mathcal{E}_{P}^{\text {univ }}\right)$ over $\mathcal{B} u n_{r, L(-m P)}$.

Proof. i) The quasiparabolic vector bundle $E^{\bullet}=\left(E^{\prime} \subseteq E\right)$ is given by the vector bundle $E$ together with a dimension $m$ quotient of the fibre $E_{P}$.

ii) $E^{\bullet}$ is also given by the vector bundle $E^{\prime}$ together with a dimension $m$ vector subspace in the fibre $E^{\prime}(P)_{P}$ of the twisted bundle $E^{\prime}(P)$. Choosing once and for all a trivialisation at $P$ for the line bundle $\mathcal{O}_{C}(P)$ identifies $E^{\prime}(P)_{P}$ and $E_{P}^{\prime}$.

In analogy with [21, the proof of our main result will use not only the above Hecke correspondence, but also the following slightly more involved comparison of Grassmannian bundles over different moduli stacks $\mathcal{B} u n_{r, L}$ :

Examples 4.3. Given $j, r_{1}, r_{2}, r_{3} \geq 1$ with $r_{1}+r_{3}=j r_{2}$ and line bundles $L_{1}, L_{2}, L_{3}$ on $C$ with $L_{1} \otimes L_{3} \cong L_{2}^{\otimes j}$, let $\mathcal{M}$ be the moduli stack of all exact sequences

$$
0 \longrightarrow E_{1} \stackrel{i}{\longrightarrow} E_{2} \otimes_{k} V \stackrel{p}{\longrightarrow} E_{3} \longrightarrow 0
$$

in which $V$ is a vector space over $k$ and $E_{1}, E_{2}, E_{3}$ are vector bundles on $C$ with $\operatorname{dim}(V)=j$ and $\operatorname{rk}\left(E_{\nu}\right)=r_{\nu}, \operatorname{det}\left(E_{\nu}\right) \cong L_{\nu}$ for $\nu=1,2,3$. More precisely, $\mathcal{M}(S)$ is the following groupoid for each $k$-scheme $S$ :

- An object consists of a rank $j$ vector bundle $\mathcal{V}$ on $S$, an object $\mathcal{E}_{\nu}$ of $\mathcal{B u n}_{r_{\nu}, L_{\nu}}(S)$ for $\nu=1,2,3$ and an exact sequence of bundles on $C \times{ }_{k} S$

$$
0 \longrightarrow \mathcal{E}_{1} \longrightarrow \mathcal{E}_{2} \otimes_{\mathcal{O}_{S}} \mathcal{V} \longrightarrow \mathcal{E}_{3} \longrightarrow 0
$$

- A morphism consists of four vector bundle isomorphisms $\lambda: \mathcal{V} \rightarrow \mathcal{V}^{\prime}$ and $\varphi_{\nu}: \mathcal{E}_{\nu} \rightarrow \mathcal{E}_{\nu}^{\prime}, \nu=1,2,3$, such that the following diagram commutes:

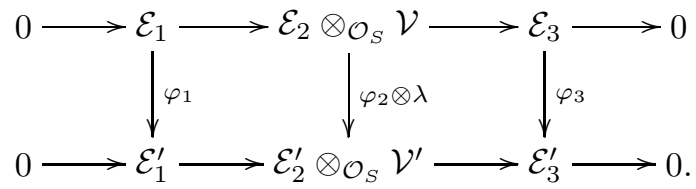




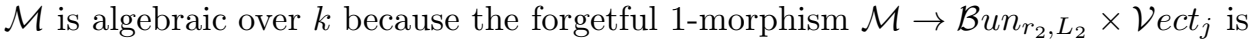
representable (by suitable open subschemes of relative Quot-schemes) and of finite type. We have a strictly commutative diagram of canonical 1-morphisms

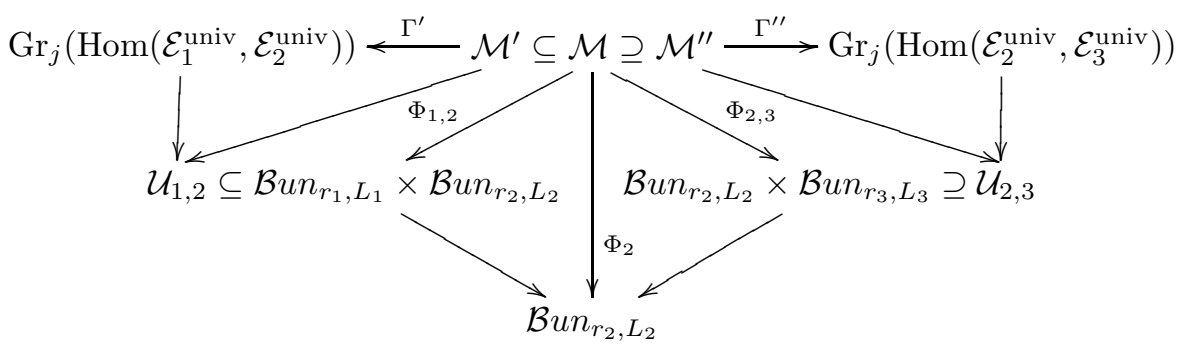

in which $\Phi_{1,2}, \Phi_{2}, \Phi_{2,3}$ are forgetful morphisms,

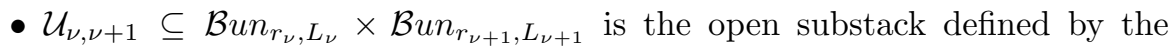
condition $\operatorname{Ext}^{1}\left(E_{\nu}, E_{\nu+1}\right)=0$,

- $\mathcal{M}^{\prime \prime} \subseteq \Phi_{2,3}^{-1}\left(\mathcal{U}_{2,3}\right)$ is the open substack where $p_{*}: V \rightarrow \operatorname{Hom}\left(E_{2}, E_{3}\right)$ is injective in (10), and $\Gamma^{\prime \prime}$ sends such a sequence to the image of $p_{*}$,

- $\mathcal{M}^{\prime} \subseteq \Phi_{1,2}^{-1}\left(\mathcal{U}_{1,2}\right)$ is the open substack where $i^{*}: V^{\text {dual }} \rightarrow \operatorname{Hom}\left(E_{1}, E_{2}\right)$ is injective in (10), and $\Gamma^{\prime}$ sends such a sequence to the image of $i^{*}$,

and the remaining four unlabelled arrows are canonical projections. We claim:

i) $\Gamma^{\prime}$ and $\Gamma^{\prime \prime}$ are open immersions.

ii) If $\operatorname{deg}\left(L_{\nu+1}\right) / r_{\nu+1}-\operatorname{deg}\left(L_{\nu}\right) / r_{\nu}>g-1$ for $\nu=1,2$ and $j$ divides $r_{\nu}, \operatorname{deg}\left(L_{\nu}\right)$ for $\nu=1,3$, then $\mathcal{M}^{\prime} \cap \mathcal{M}^{\prime \prime}$ contains a $k$-rational point.

Proof. i) An open substack of $\operatorname{Gr}_{j}\left(\operatorname{Hom}\left(\mathcal{E}_{2}^{\text {univ }}, \mathcal{E}_{3}^{\text {univ }}\right)\right)$ parameterises all linear subspaces $W \subseteq \operatorname{Hom}\left(E_{2}, E_{3}\right)$ for which the evaluation map $\epsilon_{W}: E_{2} \otimes_{k} W \rightarrow E_{3}$ is surjective. $\Gamma^{\prime \prime}$ is in fact a 1-isomorphism over $\mathcal{U}_{2,3}$ onto that open substack; its inverse sends such a linear subspace $W$ to the sequence

$$
0 \longrightarrow \operatorname{ker}\left(\epsilon_{W}\right) \longrightarrow E_{2} \otimes_{k} W \stackrel{\epsilon_{W}}{\longrightarrow} E_{3} \longrightarrow 0 .
$$

Hence $\Gamma^{\prime \prime}$ is an open immersion; considering $E_{4-\nu}^{\text {dual }}$ and $V^{\text {dual }}$ instead of $E_{\nu}$ and $V$, it follows that $\Gamma^{\prime}$ is an open immersion as well.

ii) Due to i and lemma [1.5 it suffices to show $\mathcal{M}^{\prime} \cap \mathcal{M}^{\prime \prime} \neq \emptyset$. Thus we may assume that $k=\bar{k}$ is algebraically closed.

In this case, we can choose a line bundle $\tilde{L}_{1}$ on $C$ with $\tilde{L}_{1}^{\otimes j} \cong L_{1}$; we define $\tilde{L}_{3}:=L_{2} \otimes \tilde{L}_{1}^{\text {dual }}$. Instead of $j, r_{1}, r_{2}, r_{3}$ and $L_{1}, L_{2}, L_{3}$, we consider $\tilde{j}:=1$, $r_{1} / j, r_{2}, r_{3} / j$ and $\tilde{L}_{1}, L_{2}, \tilde{L}_{3}$. We denote the resulting moduli stack by $\tilde{\mathcal{M}}$ and the analogous open substacks by $\tilde{\mathcal{M}}^{\prime} \subseteq \tilde{\mathcal{M}} \supseteq \tilde{\mathcal{M}}^{\prime \prime}$. If $\tilde{\mathcal{M}}^{\prime} \cap \tilde{\mathcal{M}}^{\prime \prime}$ has a $k$-rational point $0 \rightarrow \tilde{E}_{1} \rightarrow E_{2} \otimes_{k} \tilde{V} \rightarrow \tilde{E}_{3} \rightarrow 0$, then taking the direct sum of $j$ copies yields a $k$-rational point of $\mathcal{M}^{\prime} \cap \mathcal{M}^{\prime \prime}$. Hence it suffices to treat the case $j=1$.

In this case $j=1$, proposition 1.12 implies that the image of $\Gamma^{\prime \prime}$ described above is nonempty; hence $\mathcal{M}^{\prime \prime} \neq \emptyset$. The dual argument shows $\mathcal{M}^{\prime} \neq \emptyset$. But the forgetful 1-morphism $\mathcal{M} \rightarrow \mathcal{B u n}_{r_{1}, L_{1}} \times \mathcal{V} e c t_{1} \times \mathcal{B} u n_{r_{3}, L_{3}}$ is smooth with all fibers irreducible due to lemma 1.10 hence $\mathcal{M}$ is irreducible. This proves $\mathcal{M}^{\prime} \cap \mathcal{M}^{\prime \prime} \neq \emptyset$ here.

Corollary 4.4. In the above notation, let the vector bundle $E_{2}$ on $C$ belong to a $k$-rational point (10) of $\mathcal{M}^{\prime} \cap \mathcal{M}^{\prime \prime}$. Then the Grassmannian $\operatorname{Gr}_{j}\left(\operatorname{Hom}\left(E_{2}, \mathcal{E}_{3}^{\text {univ }}\right)\right)$ over the open substack $\emptyset \neq\left(\mathcal{U}_{2,3}\right)_{E_{2}} \subseteq \mathcal{B} u n_{r_{3}, L_{3}}$ is birational to the Grassmannian $\operatorname{Gr}_{j}\left(\operatorname{Hom}\left(\mathcal{E}_{1}^{\text {univ }}, E_{2}\right)\right)$ over the open substack $\emptyset \neq\left(\mathcal{U}_{1,2}\right)_{E_{2}} \subseteq \mathcal{B} u n_{r_{1}, L_{1}}$. 
Proof. Both Grassmannian bundles contain as a nonempty open substack the fibre of the forgetful 1-morphism $\Phi_{2}: \mathcal{M}^{\prime} \cap \mathcal{M}^{\prime \prime} \rightarrow \mathcal{B} u n_{r_{2}, L_{2}}$ over $E_{2}$.

Lemma 4.5. Suppose that $\mathcal{V}$ and $\mathcal{W}$ are two vector bundles of the same weight $w$ on an irreducible $\mathbb{G}_{m}$-gerbe $\mathcal{M}$ over $k$, and let $j \leq \operatorname{rk}(\mathcal{V})$ be given.

i) $\operatorname{Gr}_{j}(\mathcal{V} \oplus \mathcal{W})$ is birational to $\mathbb{A}^{j \cdot \mathrm{rk}(\mathcal{W})} \times \operatorname{Gr}_{j}(\mathcal{V})$.

ii) $\operatorname{Gr}_{j+\mathrm{rk}(\mathcal{W})}(\mathcal{V} \oplus \mathcal{W})$ is birational to $\mathbb{A}^{s} \times \operatorname{Gr}_{j}(\mathcal{V})$ with $s:=(\operatorname{rk}(\mathcal{V})-j) \cdot \operatorname{rk}(\mathcal{W})$.

Proof. i) Let $\emptyset \neq \mathcal{U} \subseteq \operatorname{Gr}_{j}(\mathcal{V} \oplus \mathcal{W})$ be the open substack that parameterises all the $j$-dimensional vector subspaces of $\mathcal{V}(E) \oplus \mathcal{W}(E)$ whose image $S$ in $\mathcal{V}(E)$ still has dimension $j$. Each of these subspaces is the graph of a unique linear map $S \rightarrow \mathcal{W}(E)$. Denoting by $\mathcal{S}^{\text {univ }} \subseteq p^{*} \mathcal{V}$ the universal subbundle on $p: \operatorname{Gr}_{j}(\mathcal{V}) \rightarrow \mathcal{M}$, this defines a canonical 1-isomorphism between $\mathcal{U}$ and the total space of the vector bundle $\operatorname{Hom}\left(\mathcal{S}^{\text {univ }}, p^{*} \mathcal{W}\right)$ on $\operatorname{Gr}_{j}(\mathcal{V})$. The latter has weight 0 because $\mathcal{S}^{\text {univ }}$ and $p^{*} \mathcal{W}$ both have weight $w$; hence corollary 3.8 applies to it.

ii) follows from i by applying remark 4.1 twice.

Proposition 4.6. Let $L$ be a line bundle of degree 0 on $C$. Let $\mathcal{V}$ be a vector bundle of rank $n$ and weight $w$ on an open substack $\emptyset \neq \mathcal{U} \subseteq \mathcal{B}$ un $n_{r, L}$, and let $j \leq n$.

i) If $r$ divides $w \cdot j$, then $\operatorname{Gr}_{j}(\mathcal{V})$ is birational to $\mathbb{A}^{j(n-j)} \times \mathcal{B} u n_{r, L}$.

ii) If $w \in\{0, \pm 1\}$, then $\operatorname{Gr}_{j}(\mathcal{V})$ is birational to $\mathbb{A}^{s} \times \operatorname{Gr}_{m}\left(\mathcal{E}_{P}^{\text {univ }}\right)$ for some $s$, where $m \in\{0,1, \ldots, r-1\}$ is the remainder of $w \cdot j$ modulo $r$.

iii) If $r$ and $w \cdot j$ are coprime, then $\operatorname{Gr}_{j}(\mathcal{V})$ is stably birational to $\operatorname{Gr}_{1}\left(\mathcal{E}_{P}^{\text {univ }}\right)$.

Proof. Note that $r$ divides $w \cdot n$ due to corollary $3.14 \mathrm{i}$.

i) Corollary $3.14 \mathrm{i}$ also implies that there are weight $w$ vector bundles $\mathcal{V}^{\prime}$ and $\mathcal{W}$ of rank $j$ and $n-j$ on some open substack $\emptyset \neq \mathcal{U}^{\prime} \subseteq \mathcal{B} u n_{r, L}$. According to lemma 3.10 iv, we have $\mathcal{V} \cong \mathcal{V}^{\prime} \oplus \mathcal{W}$ on some nonempty open substack of $\mathcal{U} \cap \mathcal{U}^{\prime}$; hence $\operatorname{Gr}_{j}(\mathcal{V})$ is by lemma 4.5 i birational to $\mathbb{A}^{j(n-j)} \times \operatorname{Gr}_{j}\left(\mathcal{V}^{\prime}\right) \cong \mathbb{A}^{j(n-j)} \times \mathcal{U}^{\prime}$.

ii) The case $w=0$ follows from i. Using remark 4.1 it suffices to treat the case $w=1$. Here lemma 3.10 iv allows us to assume $\mathcal{V}=\left(\mathcal{E}_{P}^{\text {univ }}\right)^{n / r}$. $\operatorname{Then} \operatorname{Gr}_{j}(\mathcal{V})$ is by lemma 4.5]ii birational to $\mathbb{A}^{s^{\prime}} \times \operatorname{Gr}_{m}\left(\mathcal{V}^{\prime}\right)$ for some $s^{\prime}$ with $\mathcal{V}^{\prime}:=\left(\mathcal{E}_{P}^{\text {univ }}\right)^{(n-j+m) / r}$. Now the claim follows from lemma $4.5 \mathrm{i}$.

iii) Let the algebraic stack $\mathcal{M}$ be the fibred product of $p: \operatorname{Gr}_{j}(\mathcal{V}) \rightarrow \mathcal{U}$ and $q: \operatorname{Gr}_{1}\left(\mathcal{E}_{P}^{\text {univ }}\right) \rightarrow \mathcal{U}$. Denoting by $\mathcal{S}^{\text {univ }} \subseteq p^{*} \mathcal{V}$ the universal subbundle, $\operatorname{det}\left(\mathcal{S}^{\text {univ }}\right)$ is a line bundle of weight $w \cdot j$ on $\operatorname{Gr}_{j}(\mathcal{V})$, whereas $p^{*} \operatorname{det}\left(\mathcal{E}_{P}^{\text {univ }}\right)$ is a line bundle of weight $r$; since these weights are coprime, there also is a line bundle $\mathcal{L}$ of weight 1 on $\operatorname{Gr}_{j}(\mathcal{V})$. Applying corollary 3.8 to $\mathcal{L}^{\text {dual }} \otimes p^{*} \mathcal{E}_{P}^{\text {univ }}$, we see that the Grassmannian bundle $\mathcal{M} \cong \operatorname{Gr}_{1}\left(p^{*} \mathcal{E}_{P}^{\text {univ }}\right)$ over $\operatorname{Gr}_{j}(\mathcal{V})$ is birational to $\mathbb{A}^{r-1} \times \operatorname{Gr}_{j}(\mathcal{V})$.

On the other hand, the universal subbundle of $q^{*} \mathcal{E}_{P}^{\text {univ }}$ is a line bundle of weight 1 on $\operatorname{Gr}_{1}\left(\mathcal{E}_{P}^{\text {univ }}\right)$; applying 3.8 as above, we see that the Grassmannian bundle $\mathcal{M} \cong \operatorname{Gr}_{j}\left(q^{*} \mathcal{V}\right)$ over $\operatorname{Gr}_{1}\left(\mathcal{E}_{P}^{\text {univ }}\right)$ is also birational to $\mathbb{A}^{j(n-j)} \times \operatorname{Gr}_{1}\left(\mathcal{E}_{P}^{\text {univ }}\right)$.

Remarks 4.7. i) Amitsur's conjecture [1 p. 40] about generic splitting fields of central simple algebras would allow to remove 'stably' in iii if $j=1$ and $n=r$. (Indeed, both Grassmannians contain neutral $\mathbb{G}_{m}$-gerbes as open substacks here, and their coarse spaces have isomorphic function fields according to the conjecture.)

ii) If $w \notin\{0, \pm 1\}$ and $r$ neither divides nor is coprime to $w \cdot j$, then the index of the Brauer class $\psi_{\operatorname{Gr}_{j}(\mathcal{V})}$ need no longer coincide with its order in the Brauer group, according to 37] for $j=1$ and to [4, 39] for general $j$. Thus there seems to be no direct generalisation of iii to this case. 


\section{Proof of the MAIN THEOREM}

Theorem 5.1. Let $L$ be a line bundle of degree $d$ on $C$. We consider an open substack $\emptyset \neq \mathcal{U}_{1} \subseteq \mathcal{B}$ un $n_{r, L}$ and a tower of Grassmannian bundles

$$
\mathcal{M}=\operatorname{Gr}_{j_{T}}\left(\mathcal{V}_{T}\right) \longrightarrow \mathcal{U}_{T} \subseteq \mathrm{Gr}_{j_{T-1}}\left(\mathcal{V}_{T-1}\right) \longrightarrow \mathcal{U}_{T-1} \ldots \mathrm{Gr}_{j_{1}}\left(\mathcal{V}_{1}\right) \longrightarrow \mathcal{U}_{1} \subseteq \mathcal{B} u n_{r, L}
$$

where $\mathcal{V}_{t}$ is a vector bundle of weight $w_{t} \in\{0, \pm 1\}$ and rank $n_{t} \geq j_{t}$ on $\mathcal{U}_{t}$ for each $t$, and $\emptyset \neq \mathcal{U}_{t+1} \subseteq \mathrm{Gr}_{j_{t}}\left(\mathcal{V}_{t}\right)$ is again an open substack.

Let $h$ denote the highest common factor of $r, d, w_{1} \cdot j_{1}, \ldots, w_{T} \cdot j_{T}$. Then $\mathcal{M}$ is birational to $\mathbb{A}^{s} \times \mathcal{B} u n_{h, L_{0}}$ for the degree 0 line bundle $L_{0}:=L(-d P)$ on $C$ with

$$
s=\operatorname{dim} \mathcal{M}-\operatorname{dim} \mathcal{B} u n_{h, L_{0}}=(g-1)\left(r^{2}-h^{2}\right)+\sum_{t} j_{t}\left(n_{t}-j_{t}\right) .
$$

Proof. i) We start with the case $h=r$, i. e. $r$ divides $d$ and $w_{1} j_{1}, \ldots, w_{T} j_{T}$. We have a 1-isomorphism $\mathcal{B} u n_{r, L_{0}} \rightarrow \mathcal{B} u n_{r, L}$ defined by sending each vector bundle $E$ on $C$ to its twist $E\left(\frac{d}{r} P\right)$; hence we may assume $d=0$ without loss of generality. Then $\operatorname{Gr}_{j_{1}}\left(\mathcal{V}_{1}\right)$ is birational to $\mathbb{A}^{j_{1}\left(n_{1}-j_{1}\right)} \times \mathcal{B} u n_{r, L_{0}}$ due to proposition 4.6] $\mathrm{i}$; replacing $k$ by the function field of $\mathbb{A}^{j_{1}\left(n_{1}-j_{1}\right)}$ and iterating, the theorem follows here.

For the remaining case $h<r$, we use induction on $r / h$. We first consider the case that $r$ divides $d$ and denote by $t \leq T$ the smallest index for which $r$ does not divide $w_{t} j_{t}$. Then $\mathcal{U}_{t}$ is birational to $\mathbb{A}^{s_{1}} \times \mathcal{B} u n_{r, L_{0}}$ for some $s_{1}$ by the previous paragraph; replacing $k$ by the function field of $\mathbb{A}^{s_{1}}$, we may assume $s_{1}=0$ and $t=1$, i. e. $\mathcal{U}_{1} \subseteq \mathcal{B}_{n_{r, L_{0}}}$ and $r$ does not divide $w_{1} \cdot j_{1}$. Here proposition 4.6 ii makes $\mathcal{U}_{2}$ birational to $\mathbb{A}^{s_{2}} \times \operatorname{Gr}_{m}\left(\mathcal{E}_{P}^{\text {univ }}\right)$ for some $s_{2}$, where $m \in\{1, \ldots, r-1\}$ is the remainder of $w_{1} \cdot j_{1}$ modulo $r$. Again, we can achieve $s_{2}=0$ by changing $k$. But then $\mathcal{U}_{2}$ is also birational to $\operatorname{Gr}_{m}\left(\left(\mathcal{E}_{P}^{\text {univ }}\right)^{\text {dual }}\right) \rightarrow \mathcal{B} u n_{r, L_{0}(m P)}$ by the examples 4.2 . Thus we may assume for the induction step that $r$ does not divide $d$.

Let $j$ be the highest common factor of $r$ and $d$; then $r / j>1$. According to elementary number theory, there is a unique integer $r_{2}$ with $r / j<r_{2}<2 r / j$ such that $r_{2} d \equiv j \bmod r$; we then define $d_{2} \in \mathbb{Z}$ by the equation

$$
(1-g) r_{2} r+r_{2} d-r d_{2}=j
$$

and put $L_{2}:=\mathcal{O}_{C}\left(d_{2} P\right)$. Now we claim that the hypotheses of 4.3 ii are satisfied with $r_{1}:=j r_{2}-r<r, r_{3}:=r$ and $L_{1}:=L^{\text {dual }}\left(j d_{2} P\right), L_{3}:=L$ :

Only the two inequalities in 4.3 ii are not obvious, but $d / r-d_{2} / r_{2}>g-1$ follows from (11), and then $d_{2} / r_{2}-d_{1} / r_{1}>g-1$ for $d_{1}:=\operatorname{deg}\left(L_{1}\right)$ follows from the computation

$$
r_{1} d_{2}-r_{2} d_{1}=\left(j r_{2}-r\right) d_{2}-r_{2}\left(j d_{2}-d\right)=r_{2} d-r d_{2}>(g-1) r_{2} r>(g-1) r_{1} r_{2} .
$$

Hence corollary 4.4 applies for some vector bundle $E_{2}$ on $C$ of rank $r_{2}$ and determinant $L_{2}$. It yields that $\operatorname{Gr}_{j}\left(\operatorname{Hom}\left(\mathcal{E}_{1}^{\text {univ }}, E_{2}\right)\right)$ over $\left(\mathcal{U}_{1,2}\right)_{E_{2}} \subseteq \mathcal{B} u n_{r_{1}, L_{1}}$ is birational to $\mathrm{Gr}_{j}$ of the vector bundle $\operatorname{Hom}\left(E_{2}, \mathcal{E}^{\text {univ }}\right)$ on $\left(\mathcal{U}_{2,3}\right)_{E_{2}} \subseteq \mathcal{B} u n_{r, L}$ whose rank is $j$ by (11); thus it is birational to $\mathcal{B u n}_{r, L}$ itself. Example 2.7] shows that $\mathcal{B}_{n_{r_{1}, L_{1}}} \cong \mathcal{B} u n_{r_{1}, L\left(-j d_{2} P\right)}$; now we can apply the induction hypothesis, observing that $r_{1}<r$ and $\operatorname{hcf}\left(r_{1}, d_{1}, j\right)=j=\operatorname{hcf}(r, d)$.

Keeping the notation of theorem 5.1 for a while, let $\emptyset \neq \mathcal{U} \subseteq \mathcal{M}$ be an open substack which is a $\mathbb{G}_{m}$-gerbe, and let $\pi: \mathcal{U} \rightarrow \mathfrak{U}$ be its coarse space.

Corollary 5.2. $\quad$ i) $\mathfrak{U}$ is birational to $\mathbb{A}^{s} \times \mathfrak{B u n}_{h, L_{0}}$ over $k$.

ii) If $h=1$, then $\mathfrak{U}$ is rational over $k$. 
iii) If $g=h=2$ and $\operatorname{char}(k)=0$, then $\mathfrak{U}$ is rational over $k$.

Proof. i) is a consequence of theorem 5.1 and lemma 2.11 iii.

ii) follows from i, since $\mathfrak{B u n} \mathfrak{n}_{1, L_{0}} \cong \operatorname{Spec}(k)$.

iii) Using i, it suffices to show $\overline{\mathfrak{B u n}}_{2, L_{0}} \cong \mathbb{P}^{3}$, or equivalently $\overline{\mathfrak{B u n}}_{2, L_{0}(2 P)} \cong \mathbb{P}^{3}$. Since $C, L$ and $P$ are defined over a finitely generated extension field of $\mathbb{Q}$, we may assume $k \subseteq \mathbb{C}$. Example 3.7 yields a determinant line bundle $\mathfrak{L}_{\text {det }}$ on $\overline{\mathfrak{B u n}}_{2, L_{0}(2 P)}$; we claim that its dual is very ample and that the resulting projective embedding is an isomorphism onto $\mathbb{P}^{3}$. To check that, we may replace $k$ by its extension field $\mathbb{C}$; then the claim holds by [31, §7] and [3, Prop. 2.5].

Corollary 5.3. With $\mathfrak{U}$ and $h$ as above, the following are equivalent for $n \in \mathbb{N}$ :

i) There is an integral, generically finite scheme $S$ of degree $n$ over $\mathfrak{U}$ that admits a Poincaré family $\mathcal{E}$ on $S$.

ii) $h$ divides $n$.

In particular, there is no Poincaré family on any open subspace of $\mathfrak{U}$ if $h \neq 1$.

Proof. Combine theorem [5.1] with corollary [3.14]ii.

Corollary 5.4. Let $\mathcal{M}$ and $h$ be as in theorem [5.1; similarly for $\mathcal{M}^{\prime}$ and $h^{\prime}$. If $h \neq h^{\prime}$, then the stacks $\mathcal{M}$ and $\mathcal{M}^{\prime}$ are not stably birational.

Proof. Suppose that $\mathbb{A}^{s} \times \mathcal{M}$ and $\mathbb{A}^{s^{\prime}} \times \mathcal{M}^{\prime}$ have a common open substack $\mathcal{U} \neq \emptyset$. We may assume that $\mathcal{U}$ is a $\mathbb{G}_{m}$-gerbe; let $\mathfrak{U}$ be its coarse space. Due to the previous corollary, $h$ and $h^{\prime}$ both coincide with the minimal degree of integral, generically finite schemes $S$ over $\mathfrak{U}$ that admit a Poincaré family; hence $h=h^{\prime}$.

Remark 5.5. Nevertheless, coarse spaces for $\mathcal{M}$ and $\mathcal{M}^{\prime}$ might be stably birational for some $h \neq h^{\prime}$, as is the case for $\operatorname{char}(k)=0, g=2$ and $h=1, h^{\prime}=2$ by corollary 5.2 All that 5.4 says is that an eventual proof of stable birationality for the coarse spaces has to contain arguments that do not carry over to the stacks.

Corollary 5.6. Let $\mathcal{M}$ and $h$ be as above; let $n \in \mathbb{N}$ and $w \in \mathbb{Z}$ be given. Then there exists an open substack $\emptyset \neq \mathcal{U} \subseteq \mathcal{M}$ and a vector bundle $\mathcal{V}$ of rank $n$ and weight $w$ on $\mathcal{U}$ if and only if $h$ divides $w \cdot n$.

Proof. This follows directly from theorem 5.1 lemma 2.8 and corollary $3.14 \mathrm{i}$.

Example 5.7. Let $D \subset C(k)$ be a finite set of points on our curve $C$. We consider vector bundles $E$ on $C$ with fixed rank $r$ and degree $d$, endowed with a quasiparabolic structure with fixed multiplicities $m_{1}(P), \ldots, m_{l}(P)$ at each point $P \in D$. Let $\mathfrak{P a r}_{r, d} \frac{m}{D}$ be their coarse moduli scheme for some given weights $\alpha_{1}, \ldots, \alpha_{l}$, as introduced in [30. Then the above implies in particular:

i) All fibers of det $: \mathfrak{P a r}_{r, d}^{\frac{m}{/ D}} \rightarrow \operatorname{Pic}^{d}(C)$ are rational if the highest common factor $h$ of $r, d$ and all multiplicities $m_{l}(P)$ is 1. (This generalises [5].)

ii) There is a Poincaré family on some nonempty open subscheme of $\mathfrak{P a r}_{r, d} / D$ if and only if $h=1$.

Example 5.8. Concerning the coarse moduli scheme $\mathfrak{B u n}_{r, L}$ of vector bundles with fixed determinant $L$ of degree $d$, the above reproves the rationality theorem of King and Schofield 21] and the non-existence theorem for Poincaré families due to Ramanan [34. Moreover, we get the following additional information: 
i) There is an integral, generically finite scheme of degree $n$ over $\mathfrak{B u n} \mathfrak{n}_{r, L}$ with a Poincaré family if and only if $\operatorname{hcf}(r, d)$ divides $n$.

ii) For a fixed vector bundle $F$ on $C$, the minimal dimension of $\operatorname{dim}_{k} \operatorname{Hom}(F, E)$ for rank $r$, determinant $L$ vector bundles $E$ on $C$ is a multiple of $\operatorname{hcf}(r, d)$. (ii follows from [5.6] and example1.11ii. For $F$ generic, it also follows from the the-

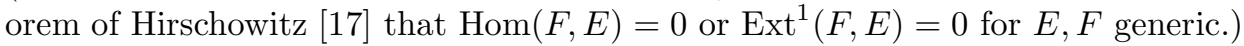

Example 5.9. Given a closed subscheme $\emptyset \neq D \varsubsetneqq C$, we consider rank $r$ vector bundles $E$ on $C$ with $\operatorname{det}(E) \cong L$, endowed with a level structure at $D$, i.e. with a nonzero morphism $E \rightarrow \mathcal{O}_{D}^{r}$. Let $\mathfrak{L e v}_{r, L}^{D}$ be the coarse moduli scheme of such $E$ with level structure which are stable in the sense of [38. Then $\mathfrak{L e v}_{r, L}^{D}$ is rational.

Proof. Let $\mathcal{V}$ be the vector bundle of weight -1 on $\mathcal{B} u n_{r, L}$ whose fibre at any point $[E]$ is $\operatorname{Hom}_{\mathcal{O}_{C}}\left(E, \mathcal{O}_{D}^{r}\right)$. Then $\mathfrak{L e v}_{r, L}^{D}$ is the coarse space associated to an open substack of $\operatorname{Gr}_{1}(\mathcal{V})$; hence it is rational by corollary 5.2 ii above.

More generally, the above results can be applied in a similar way to stable pairs on $C$ in the sense of Huybrechts and Lehn [19, and to other sorts of augmented bundles on $C$ in the sense of [7; we mention:

Example 5.10. A coherent system $(E, V)$ of type $(r, d, n)$ consists of a rank $r$, degree $d$ vector bundle $E$ on $C$ and an $n$-dimensional vector subspace $V \subseteq \mathrm{H}^{0}(E)$, cf. [27, 33] or 22. Assuming $k=\mathbb{C}, n<r, 0<d$ and $n \leq d+(r-n)(g-1)$, let $\mathfrak{C o h}_{r, d}^{n}$ be the coarse moduli scheme of such coherent systems which are $\alpha$-stable for the largest possible value $\alpha=d /(r-n)-\epsilon$ of the stability parameter $\alpha \in \mathbb{R}[$ ], §3]. Then $\mathfrak{C o h}_{r, d}^{n}$ is birational to $\mathbb{A}^{s} \times \mathfrak{B u n}_{h, 0}$ for $h:=\operatorname{hcf}(r, d, n)$ and some $s \in \mathbb{N}$.

Proof. Let $\mathcal{V}$ be the vector bundle of weight -1 on $\mathcal{B} u n_{r-n, d}^{\text {stab }}$ whose fibre at any point $\left[E^{\prime}\right]$ is $\operatorname{Ext}^{1}\left(E^{\prime}, \mathcal{O}_{C}\right)$; note that $\operatorname{Hom}\left(E^{\prime}, \mathcal{O}_{C}\right)=0$ due to the stability of $E^{\prime}$, so $\mathcal{V}$ is indeed a vector bundle of rank $d+(r-n)(g-1) \geq n$. The coarse space of the Grassmannian $\operatorname{Gr}_{n}(\mathcal{V})$ over $\mathcal{B} u n_{r-n, d}^{\text {stab }}$ is birational to $\mathfrak{C o h}_{r, d}^{n}$ according to the proof of [8, Thm. 4.5]; now apply corollary [5.2 i over the function field of $\operatorname{Pic}^{d}(C)$.

Example 5.11. Let $\mathfrak{B N}_{r, d}^{n} \subseteq \mathfrak{B u n}_{r, d}$ be the Brill-Noether locus of vector bundles $E$ on $C$ with $\operatorname{dim}_{k} \mathrm{H}^{0}(E) \geq n$. Assume $n=1$ or that $C$ is a Petri curve and $n \in\{2,3\}$. If $k=\mathbb{C}, n<r$ and $0<d<r(g-1)+n$, then $\mathfrak{B N}_{r, d}^{n}$ is birational to $\mathbb{A}^{s} \times \mathfrak{B u n}_{h, 0}$ for $h:=\operatorname{hcf}(r, d, n)$ and some $s \in \mathbb{N}$ (or empty if $n=3, r=4, d=1$ and $g=2$ ).

Proof. Here $\mathfrak{B N}_{r, d}^{n}$ is birational to $\mathfrak{C o h}_{r, d}^{n}$, according to [9, Section 11.2].

There seems to be no easy generalisation of theorem 5.1 to weights $w \notin\{0, \pm 1\}$, mainly because an analogue of the Hecke correspondence 4.2 is missing; cf. also remark 4.7 But at least, we have the following:

Corollary 5.12. Let $L$ be a line bundle of degree d on $C$, let $\mathcal{V}$ be a vector bundle of weight $w$ on an open substack $\emptyset \neq \mathcal{U} \subseteq \mathcal{B} u n_{r, L}$, and let $j \leq \operatorname{rk}(\mathcal{V})$. If $r, d$ and $w \cdot j$ have no common factor $\neq \pm 1$, then $\operatorname{Gr}_{j}(\mathcal{V})$ is stably birational to $\mathcal{B}$ un $n_{1, L} \cong \mathrm{B} \mathbb{G}_{m}$.

Proof. Replacing $r$ by $\operatorname{hcf}(r, d)$ and $k$ by a rational function field over $k$, theorem 5.1 allows us to assume $d=0$ without loss of generality. This case follows from proposition 4.6 iii and theorem 5.1 again. 
N. HOFFMANN

Example 5.13. Over $k=\mathbb{C}$, let $\rho: \mathrm{GL}(r) \rightarrow \mathrm{GL}(V)$ be an algebraic representation that maps the center $\mathbb{G}_{m} \subseteq \mathrm{GL}(r)$ to the center $\mathbb{G}_{m} \subseteq \mathrm{GL}(V)$ with weight $w \in \mathbb{Z}$. Let $M$ be a line bundle on $C$. We consider vector bundles $E$ of rank $r$ and degree $d$ on $C$ which are decorated in the sense of A. Schmitt 36, i. e. endowed with an element of $\mathbb{P} \operatorname{Hom}\left(E_{\rho}, M\right)$ where $E_{\rho}$ is the vector bundle on $C$ with fibre $V$ associated to the GL $(r)$-bundle given by $E$.

We fix a stability parameter $\delta>0$ and choose $M$ of sufficiently large degree such that the resulting coarse moduli scheme $\mathfrak{D} \mathfrak{e c}_{r, d}^{M}$ of $\delta$-stable decorated vector bundles is irreducible by [36, Thm. 3.5]. If $r, d$ and $w$ have no common factor $\neq \pm 1$, then the generic fibre of det $: \mathfrak{D e c}_{r, d}^{M} \rightarrow \operatorname{Pic}^{d}(C)$ is stably rational.

\section{REFERENCES}

[1] S. Amitsur. Generic splitting fields of central simple algebras. Ann. of Math. (2), 62:8-43, 1955.

[2] M. Artin. Brauer-Severi varieties. In Brauer groups in ring theory and algebraic geometry (Antwerp 1981), pages 194-210. Springer-Verlag, Berlin, 1982.

[3] A. Beauville. Fibrés de rang 2 sur une courbe, fibré déterminant et fonctions thêta. Bull. Soc. Math. France, 116(4):431-448 (1989), 1988.

[4] A. Blanchet. Function fields of generalized Brauer-Severi varieties. Comm. Algebra, 19(1):97$118,1991$.

[5] H. U. Boden and K. Yokogawa. Rationality of moduli spaces of parabolic bundles. J. London Math. Soc. (2), 59(2):461-478, 1999.

[6] N. Bourbaki. Commutative algebra. Chapters 1-7. Elements of Mathematics. Springer-Verlag, Berlin, 1989.

[7] S. Bradlow, G. Daskalopoulos, O. García-Prada, and R. Wentworth. Stable augmented bundles over Riemann surfaces. In Vector bundles in algebraic geometry (Durham 1993), pages 15-67. Cambridge Univ. Press, Cambridge, 1995.

[8] S. Bradlow and O. García-Prada. An application of coherent systems to a Brill-Noether problem. J. Reine Angew. Math., 551:123-143, 2002.

[9] S. Bradlow, O. García-Prada, V. Muñoz, and P. Newstead. Coherent systems and BrillNoether theory. Internat. J. Math., 14(7):683-733, 2003.

[10] D. K. Faddeev. The group of divisor classes on some algebraic curves. Soviet Math. Dokl., 2:67-69, 1961.

[11] G. Faltings. Vector bundles on curves. Lectures held in Bonn, 1995.

[12] J. Giraud. Cohomologie non abélienne. Springer-Verlag, Berlin, 1971.

[13] A. Grothendieck. EGA III: Étude cohomologique des faisceaux cohérents. Inst. Hautes Études Sci. Publ. Math., 11/17, 1961/63.

[14] A. Grothendieck. EGA IV: Étude locale des schémas et des morphismes de schémas. Inst. Hautes Études Sci. Publ. Math., 20/24/28/32, 1964-67.

[15] A. Grothendieck et al. SGA IV: Théorie des topos et cohomologie étale des schémas, volume 269, 270, 305 of Lecture Notes in Mathematics. Springer-Verlag, Berlin, 1972/73.

[16] J. Heinloth. Über den Modulstack der Vektorbündel auf Kurven. Diploma thesis, University of Bonn, 1998.

[17] A. Hirschowitz. Problèmes de Brill-Noether en rang supèrieur. http://math.unice.fr/ ah/math/Brill/.

[18] N. Hoffmann. Moduli stacks of vector bundles on curves and the King-Schofield rationality proof. preprint, available at www.arXiv.org.

[19] D. Huybrechts and M. Lehn. Stable pairs on curves and surfaces. J. Algebraic Geom., 4(1):67104, 1995.

[20] D. Huybrechts and M. Lehn. The geometry of moduli spaces of sheaves. Friedr. Vieweg \& Sohn, Braunschweig, 1997.

[21] A. King and A. Schofield. Rationality of moduli of vector bundles on curves. Indag. Math. (N.S.), 10(4):519-535, 1999.

[22] A. D. King and P. E. Newstead. Moduli of Brill-Noether pairs on algebraic curves. Internat. J. Math., 6(5):733-748, 1995. 
[23] F. Knop, H. Kraft, and T. Vust. The Picard group of a G-variety. In Algebraische Transformationsgruppen und Invariantentheorie, pages 77-87. Birkhäuser, Basel, 1989.

[24] F. F. Knudsen and D. Mumford. The projectivity of the moduli space of stable curves. I. Preliminaries on "det" and "Div". Math. Scand., 39(1):19-55, 1976.

[25] D. Knutson. Algebraic spaces. Springer-Verlag, Berlin, 1971. Lecture Notes in Mathematics, Vol. 203.

[26] G. Laumon and L. Moret-Bailly. Champs algébriques. Springer-Verlag, Berlin, 2000.

[27] J. Le Potier. Systèmes cohérents et structures de niveau, volume 214 of Astérisque. Société Mathématique de France, Paris, 1993.

[28] M. Lieblich. Moduli of twisted sheaves. preprint math.AG/0411337

[29] M. Lieblich. Twisted sheaves and the period-index problem. preprint math.AG/0511244

[30] V. B. Mehta and C. S. Seshadri. Moduli of vector bundles on curves with parabolic structures. Math. Ann., 248(3):205-239, 1980.

[31] M. S. Narasimhan and S. Ramanan. Moduli of vector bundles on a compact Riemann surface. Ann. of Math. (2), 89:14-51, 1969.

[32] P. E. Newstead. Introduction to moduli problems and orbit spaces, volume 51 of TIFR Lectures on Mathematics and Physics. Tata Institute of Fundamental Research, Bombay, 1978.

[33] N. Raghavendra and P. Vishwanath. Moduli of pairs and generalized theta divisors. Tohoku Math. J. (2), 46(3):321-340, 1994.

[34] S. Ramanan. The moduli spaces of vector bundles over an algebraic curve. Math. Ann., 200:69-84, 1973.

[35] B. Russo and M. Teixidor i Bigas. On a conjecture of Lange. J. Algebraic Geom., 8(3):483496, 1999.

[36] A. Schmitt. A universal construction for moduli spaces of decorated vector bundles over curves. Transform. Groups, 9:167-209, 2004.

[37] A. Schofield and M. Van den Bergh. The index of a Brauer class on a Brauer-Severi variety. Trans. Amer. Math. Soc., 333(2):729-739, 1992.

[38] C. S. Seshadri. Fibrés vectoriels sur les courbes algébriques, volume 96 of Astérisque. Société Mathématique de France, Paris, 1982.

[39] A. Wadsworth. The index reduction formula for generic partial splitting varieties. Comm. Algebra, 21(4):1063-1070, 1993.

Mathematisches Institut der Universität, Bunsenstr. 3-5, 37073 Göttingen, Germany

Current address: School of Mathematics, Tata Institute of Fundamental Research, Homi Bhabha Road, Mumbai 400005, India

E-mail address: hoffmann@uni-math.gwdg.de 